\title{
Quality of vitamin K antagonist control and outcomes in atrial fibrillation patients: a meta-analysis and meta-regression
}

Elizabeth S Mearns ${ }^{1,2}$, C Michael White ${ }^{1,2}$, Christine G Kohn ${ }^{1,2}$, Jessica Hawthorne ${ }^{1}$, Ju-Sung Song ${ }^{1}$, Joy Meng ${ }^{1}$, Jeff R Schein ${ }^{3}$, Monika K Raut ${ }^{3}$ and Craig I Coleman ${ }^{1,2^{*}}$

\begin{abstract}
Background: Atrial fibrillation (AF) patients frequently require anticoagulation with vitamin $\mathrm{K}$ antagonists (VKAs) to prevent thromboembolic events, but their use increases the risk of hemorrhage. We evaluated time spent in therapeutic range (TTR), proportion of international normalized ratio (INR) measurements in range (PINRR), adverse events in relation to INR, and predictors of INR control in AF patients using VKAs.

Methods: We searched MEDLINE, CENTRAL and EMBASE (1990-June 2013) for studies of AF patients receiving adjusted-dose VKAs that reported INR control measures (TTR and PINRR) and/or reported an INR measurement coinciding with thromboembolic or hemorrhagic events. Random-effects meta-analyses and meta-regression were performed.

Results: Ninety-five articles were included. Sixty-eight VKA-treated study groups reported measures of INR control, while 43 studies reported an INR around the time of the adverse event. Patients spent 61\% (95\% Cl, 59-62\%), 25\% (95\% Cl, 23-27\%) and 14\% (95\% Cl, 13-15\%) of their time within, below or above the therapeutic range. PINRR assessments were within, below, and above range 56\% (95\% Cl, 53-59\%), 26\% (95\% Cl, 23-29\%) and 13\% (95\% Cl, 11-17\%) of the time. Patients receiving VKA management in the community spent less TTR than those managed by anticoagulation clinics or in randomized trials. Patients newly receiving VKAs spent less TTR than those with prior VKA use. Patients in Europe/United Kingdom spent more TTR than patients in North America. Fifty-seven percent $(95 \% \mathrm{Cl}$, $50-64 \%)$ of thromboembolic events and $42 \%(95 \% \mathrm{Cl}, 35-51 \%)$ of hemorrhagic events occurred at an INR $<2.0$ and $>3.0$, respectively; while $56 \%(95 \% \mathrm{Cl}, 48-64 \%)$ of ischemic strokes and $45 \%$ of intracranial hemorrhages (95\% Cl, 29-63\%) occurred at INRs $<2.0$ and $>3.0$, respectively.
\end{abstract}

Conclusions: Patients on VKAs for AF frequently have INRs outside the therapeutic range. While, thromboembolic and hemorrhagic events do occur patients with a therapeutic INR; patients with an INR $<2.0$ make up many of the cases of thromboembolism, while those $>3.0$ make up many of the cases of hemorrhage. Managing anticoagulation outside of a clinical trial or anticoagulation clinic is associated with poorer INR control, as is, the initiation of therapy in the VKA-naïve. Patients in Europe/UK have better INR control than those in North America.

Keywords: Vitamin K antagonists, Atrial fibrillation, International normalized ratio, Anticoagulation

\footnotetext{
* Correspondence: Craig.Coleman@hhchealth.org

${ }^{1}$ Department of Pharmacy Practice, University of Connecticut School of

Pharmacy, 69 N Eagleville Road, Storrs, CT 06269-3092, USA

${ }^{2}$ Hartford Hospital Division of Cardiology, 80 Seymour Street, Hartford, CT

06102-5037, USA

Full list of author information is available at the end of the article
}

C Biomed Central (c) 2014 Mearns et al.; licensee BioMed Central Ltd. This is an Open Access article distributed under the terms of the Creative Commons Attribution License (http://creativecommons.org/licenses/by/4.0), which permits unrestricted use, distribution, and reproduction in any medium, provided the original work is properly credited. The Creative Commons Public Domain Dedication waiver (http://creativecommons.org/publicdomain/zero/1.0/) applies to the data made available in this article, unless otherwise stated. 


\section{Background}

Atrial fibrillation (AF) is the most common sustained cardiac arrhythmia worldwide and increases the risk of ischemic stroke by nearly 5 -fold $[1,2]$. Studies have demonstrated that adjusted-dose vitamin $\mathrm{K}$ antagonists (VKAs) significantly decrease the risk of stroke in AF patients versus placebo or aspirin therapy $[3,4]$. However, VKA use can be challenging given the narrow therapeutic international normalized ratio (INR) range, requirement for periodic INR monitoring, high inter-patient variability in response, numerous drug and food interactions and risks related to non-adherence [5].

In previous meta-analyses [6-9] the quality of anticoagulation control with VKAs has proven to be poor; with an estimated time spent in the therapeutic INR range (TTR) between 55\% and 64\%. Numerous study-level factors (e.g., VKA dosing setting) have been shown to be an important determinant of the quality of INR control. Thromboembolic events may occur more frequently at an INR $<2.0$ and major hemorrhagic events at an INR $>3.0$ [10]. No previous meta-analyses have examined INR control in VKA-experienced as compared to naïve patients as a study-level factor, not all analyses have looked at AF alone, and few have assessed the percentage of INRs in therapeutic range (PINRR) as a quality measure of INR control. Moreover, there has been a substantive increase in the number of studies assessing INRs in patients with AF receiving VKAs in the past few years, lending more power and validity to a systematic assessment of INR control being conducted now.

The primary objective of this systematic review with meta-analyses and meta-regression analyses was to assess VKA INR control in AF patients and the effect of selected study-level factors (including prior VKA treatment experience) on TTR and the PINRR, and to evaluate the relationship between the proportion of VKA-associated hemorrhagic and thromboembolic events that occurred when the INR was above or below the therapeutic range.

\section{Methods}

\section{Study selection}

A systematic review of MEDLINE, CENTRAL and EMBASE (from 1990 through June 2013) was performed. Our search strategy for Medline is provided in the Additional file 1. Two investigators reviewed all potentially relevant articles independently, with disagreement resolved by a third investigator. Studies were selected for inclusion on the basis of the following criteria: English full-text randomized controlled trials (RCT), prospective cohort studies or retrospective analyses; contained $\geq 50$ patients in each treatment group; conducted in adult patients ( $\geq 18$ years of age) receiving dose-adjusted VKA with AF as their primary reason for anticoagulation; and INR control reported as TTR, PINRR or report an
INR measurement at (within 48 hours of the event) or near (more than 48 hours from the event) the time of a hemorrhagic or thromboembolic event. Studies were excluded if the duration of study was $<3$ months, the target INR range was other than 2.0 to 3.0, or patient population overlapped with another study. Manual backward citation tracking of references from identified studies and review articles was also performed to identify additional relevant studies.

\section{Data abstraction}

Two investigators used a common data abstraction tool, but independently abstracted all data. If a disagreement arose it was resolved by a third investigator. The following study-level information was obtained: author identification, year of publication (1990-2000, 2001-2007 or 2008-2013), whether patients were VKA-naïve $(<30 \%$ of the population receiving a VKA prior to entering the study) or experienced ( $>70 \%$ of the population receiving a VKA prior to entering the study), geographic location of the study (Europe/ United Kingdom (UK), Asia, North America, multinational or other), duration of VKA treatment, specific VKA used, interpolation method, whether patients were utilizing VKA self-management to monitor INR control and the study setting (anticoagulation clinic, RCT, or community/standard practice). The setting was designated using the following definitions: an anticoagulation clinic, if the study took place in an anticoagulation clinic or if the stated role of the study clinicians in patient care was limited to managing anticoagulation; a randomized trial if random allocation was employed to assign subjects to receive warfarin or another non-warfarin therapy; and all others were classified as community practice.

Measures of INR control and outcomes were abstracted from each study including TTR, time spent above range, time spent below range, PINRR, proportion of INR measurements above and below therapeutic range and clinical outcomes of major hemorrhage and thromboembolic events. Major hemorrhages included intracranial hemorrhage $(\mathrm{ICH})$ and extracranial bleeding (bleeding requiring hospitalization, blood transfusion or surgical treatment or occurring at a critical anatomical location). All thromboembolic events were abstracted including ischemic stroke, systemic emboli, venous thromboembolism and myocardial infarction. INR measurements at or near each thromboembolic and hemorrhagic event were abstracted when reported.

\section{Statistical analysis}

TTR and PINRR for each study group, as well as the time/proportions below and above range for these measures were expressed as an incidence density using a person-time approach. The numerator was calculated as the proportion of time that the group spent within, below or above the 
INR range or proportion of INR measurements in, below, or above range multiplied by person-years of follow-up. The denominator was the total person-years of observation for each study group (or the mean/median observation time multiplied by the number of patients in each study group, if person-years of follow-up for the VKA $\operatorname{arm}(\mathrm{s})$ in a study was not reported). Ninety-five percent confidence intervals (CIs) were calculated for each incidence density using the Wilson score method without continuity correction. For the purposes of these analyses, all studies were pooled using a random-effects model.

In order to determine how study-level factors influenced TTR or PINRR, both subgroup and meta-regression analyses were conducted. Meta-regression analysis allows evaluating the effect of any given influencing factor independent of the effect of other aspects. A multiple linear mixed method model using both random- and fixed-effects was utilized for meta-regression, which was weighted by the inverse of the variance of TTR or PINRR. Fixed-effects were assumed for study-level factors, including the following covariates: prior experience with VKAs (naïve, experienced, mixed/not reported), study design (community, anticoagulation clinic, RCT), study year (from 19902000 and 2001-7, 2008-2013), use of self-management or not, interpolation method (linear or other), geographic region (North America [United States and Canada], Europe/ UK, Asia, multinational, other) and duration of VKA treatment ( $<1$ year, $\geq 1$ year). No hierarchy was used in the model for these covariates.

The weighted proportion of thromboembolic and hemorrhagic events occurring outside of the INR range for each study group was also calculated. For this analysis, the numerator was the number of thromboembolic events occurring below an INR of 2.0 or the number of hemorrhagic events above an INR of 3.0. The denominators were the total number of thrombotic or hemorrhagic events for each study group. Ninety-five percent CIs were calculated for each proportion using the Wilson score method without continuity correction. Again, all studies were pooled using a random-effects model. Since not all studies measured INR values at the exact time of adverse outcomes, we conducted two separate analyses, one pooling studies regardless of the elapsed time between the INR measurement and the adverse event; and a second (sensitivity analysis) including only those studies specifically stating an INR was measured within 48 hours of the event.

For all meta-analyses, statistical heterogeneity was determined using the $\mathrm{I}^{2}$ statistic (with an $\mathrm{I}^{2}>50 \%$ signifying an important degree of statistical heterogeneity) and publication bias was assessed using the Egger's weighted regression statistic (with a $\mathrm{p}<0.05$ suggesting a higher likelihood of publication bias) and through review of funnel plots (scatterplots of effect size against standard error, where the each dot represents a study effect estimate and the vertical line represents the pooled effect). Statistical analyses were performed using StatsDirect version 2.7.6 (StatsDirect Ltd., Cheshire, England), SAS, version 9.2 (SAS Institute Inc., Cary, NC), and SPSS 15.0 for Windows (SPSS, Inc, Chicago, IL).

\section{Results}

We identified 5,326 citations, of which 5,231 were excluded (Figure 1). Ninety-five articles met our inclusion and exclusion criteria [11-103]. Sixty-eight studies reported measures of INR control representing 87 VKA study arms, and 43 studies reported an INR at or near the time of the adverse event (16 studies reported both and were included in both analyses).

Of the 87 VKA study groups that reported a measure of INR control, 17 reported data on patients with no prior VKA exposure whereas 47 enrolled patients who were VKA-experienced and 23 did not report prior VKA exposure data or had mixed enrollment. Twenty-eight VKA groups were from RCTs, 27 were conducted in an anticoagulation clinic setting, and 32 were in a community practice setting (Table 1). Thirty-nine VKA study groups were recruited in Europe/UK, 5 from Asia, 13 were multinational, 1 from Israel and 29 from North America. The mean age ranged from 62 to 92 years (median, 72 years), the number of VKA-treated patients ranged from 55 to 11,770 (median, 249), and the personyears of VKA therapy ranged from 17 to 30,188 (median, 399). Of the 43 identified studies that reported an INR with an adverse event, 16 were RCTs, 14 were conducted in an anticoagulation clinic, and 13 were from community practice with the number of VKA-treated patients ranged from 55 to 9,217 (median, 288) (Table 1).

For the first meta-analysis, 78 out of 87 (90\%) study groups reported a TTR and $55(63 \%)$ reported the time spent below and above range; 22 of 87 (25\%) study groups reported a PINRR and 21 (24\%) reported the percent of INR measures below and above range (Table 2). Thirteen study groups (15\%) reported both TTR and PINRR. Overall, patients spent $61 \%$ of their TTR (95\% CI, 59-62\%), 25\% (95\% CI, 23-27\%) below, and 14\% (95\% CI, 13-15\%) above range. Similarly for PINRR, $56 \%$ of INR measurements were in the therapeutic range (95\% CI, 53-59\%), $26 \%$ (95\% CI, 23-29\%) below and 13\% (95\% CI, 11-17\%) above range. Statistical heterogeneity was observed to be high between the groups included in these analyses $\left(\mathrm{I}^{2} \geq 97 \%\right)$. The likelihood of publication bias appeared low for TTR, time above range, PINRR, and proportion of INR measurements below and above range. However, publication bias was deemed more likely for the time below range analysis (Egger's $\mathrm{p}=0.03$ ) (Additional file 1 ).

Upon meta-regression to determine how study-level factors influenced TTR, community VKA management was associated with less time in range than therapy 
managed in an anticoagulation clinic or RCT setting, European/UK study patients spent more time in range than North American study patients, and the VKA-naïve (and mixed/not reported) spent less time in range than VKA-experienced patients (Table 3). For PINRR, no studylevel factor was found to be significantly different than the referent upon meta-regression $(\mathrm{p}>0.05)$ (Table 4).

In the second meta-analysis, 30 of 43 studies (70\%) reported an INR measure with thromboembolic events, 21 (49\%) reported an INR with ischemic stroke, 32 (74\%) reported INRs with major hemorrhage, and 16 (37\%) reported an INR with ICH. INR data was incomplete in $58 \%$ of the studies that reported adverse events; not all events were reported with an INR measure. Thirty percent of the studies used previously reported INRs, allowing INR measures from 3 days up to 12 days prior to be considered as temporally related to the adverse event.

Overall, $57 \%$ of the thromboembolic events occurred at an INR $<2.0$ (95\% CI, 50-64\%) and $42 \%$ of hemorrhagic events occurred at an INR $>3.0$ (95\% CI, $35-51 \%)$ (Figures 2 and 3). A high degree of heterogeneity was present for studies that reported thromboembolic and hemorrhagic events $\left(\mathrm{I}^{2}=80 \%\right.$ and $77 \%$ respectively); however, the presence of publication bias was deemed low (Egger's $\mathrm{p}=0.31$ and $\mathrm{p}=0.69$, respectively) (Additional file 1). When ischemic stroke and $\mathrm{ICH}$ were evaluated separately from other events, we found that $56 \%$ of ischemic strokes (95\% CI, $48-64 \%$ ) and $45 \%$ of ICHs (95\% CI, 
Table 1 Demographics of included studies

\begin{tabular}{|c|c|c|c|c|c|c|c|c|c|}
\hline Study, Year & Study design/setting & VKA-treated N & Mean age & VKA experience, $\%$ & Geographic region & $\begin{array}{l}\text { Interpolation } \\
\text { method }\end{array}$ & $\begin{array}{c}\text { Self - } \\
\text { management }\end{array}$ & $\begin{array}{l}\text { Duration of therapy, } \\
\text { months (Patient-years) }\end{array}$ & $\begin{array}{c}\text { VKA } \\
\text { studied }\end{array}$ \\
\hline Abdelhafiz 2004 [11] & PD/AC clinic & 402 & 72 & $0 \%$ & Europe & Linear & $N$ & $19(637)$ & W \\
\hline Abdelhafiz (<75) 2008 [12] & \multirow{2}{*}{ PD/AC clinic } & 203 & 64 & \multirow{2}{*}{$0 \%$} & \multirow{2}{*}{ Europe } & \multirow{2}{*}{ Linear } & \multirow{2}{*}{ N } & $19(321)$ & \multirow{2}{*}{ W } \\
\hline Abdelhafiz ( $\geq 75) 2008$ [12] & & 199 & 80 & & & & & $19(315)$ & \\
\hline Akdeniz 2005 [13] & PD/Community & 208 & 66 & $N R$ & Turkey & - & - & - & W \\
\hline Albers 2005 [14] & RCT & 1962 & 72 & $85 \%$ & N. America & Linear & N & $20(3270)$ & W \\
\hline Anderson 2004 [15] & $\mathrm{RD} / \mathrm{AC}$ clinic & 87 & NR & $100 \%$ & N. America & NR & N & $12(87)$ & W \\
\hline Ansell (Italy) 2007 [16] & $\mathrm{RD} / \mathrm{AC}$ clinic & 177 & 72 & \multirow{5}{*}{$100 \%$} & \multirow{5}{*}{ Multinational } & \multirow{5}{*}{ Linear } & \multirow{5}{*}{ N } & $12(177)$ & W \\
\hline Ansell (Spain) 2007 [16] & $\mathrm{RD} / \mathrm{AC}$ clinic & 218 & 72 & & & & & $12(218)$ & A \\
\hline Ansell (US) 2007 [16] & RD/Community & 686 & 75 & & & & & $12(686)$ & W \\
\hline Ansell (Canada) 2007 [16] & RD/Community & 152 & 74 & & & & & $12(152)$ & W \\
\hline Ansell (France) 2007 [16] & RD/Community & 278 & 73 & & & & & $12(278)$ & $\mathrm{F}$ \\
\hline Aronow 1999 [17] & PD/Community & 125 & 83 & NR & N. America & $N R$ & N & $34(354)$ & W \\
\hline Boulanger 2006 [18] & RD/Community & 6454 & 68 & $65 \%$ & N. America & Linear & $N$ & $12(6454)$ & W \\
\hline Burton (<75) 2006 [19] & \multirow{2}{*}{ RD/Community } & 260 & \multirow{2}{*}{ NR } & \multirow{2}{*}{ Mixed } & \multirow{2}{*}{ Europe } & \multirow{2}{*}{ Linear } & \multirow{2}{*}{ N } & $25(539)$ & \multirow{2}{*}{ W } \\
\hline Burton (>75) 2006 [19] & & 341 & & & & & & $15(414)$ & \\
\hline Cafolla (Manual) 2011 [20] & RD/Community & 576 & NR & $100 \%$ & Europe & Linear & N & $8(384)$ & $A, W$ \\
\hline Cafolla 2012 [21] & $\mathrm{PD} / \mathrm{AC}$ clinic & 57 & 85 & $100 \%$ & Europe & Linear & N & $18(86)$ & W \\
\hline Cheung 2005 [22] & RD/Community & 555 & 70 & $100 \%$ & Asia & - & $\mathrm{N}$ & 19 (893) & W \\
\hline Chung 2011 [23] & $\mathrm{RCT}$ & 75 & 65 & $55 \%$ & Asia & Linear & $N$ & $3(19)$ & W \\
\hline Connolly 1991 [24] & $\mathrm{RCT}$ & 187 & 68 & $0 \%$ & N. America & Linear & N & $15(234)$ & W \\
\hline Connolly 2006 [25] & $\mathrm{RCT}$ & 3371 & 70 & $78 \%$ & N. America & Other & N & $15(4214)$ & $N R$ \\
\hline Copland 2001 [26] & RD/AC Clinic & 328 & 70 & $100 \%$ & UK & - & $\mathrm{N}$ & $21(458)$ & W \\
\hline Currie (stable) 2005 [27] & \multirow{2}{*}{ RD/Community } & 787 & 74 & \multirow{2}{*}{$100 \%$} & \multirow{2}{*}{ Europe } & \multirow{2}{*}{ Linear } & \multirow{2}{*}{ N } & $35(2282)$ & \multirow{2}{*}{ W } \\
\hline Currie (unstable) 2005 [27] & & 726 & 78 & & & & & $35(2105)$ & \\
\hline Dentali 2012 [28] & PD/AC Clinic & 221 & 75 & 100 & Europe & - & N & $3(663)$ & W \\
\hline DiMarco 2005 [29] & $\mathrm{RCT}$ & NR & 70 & $N R$ & N. America & - & N & $42(-)$ & W \\
\hline Dimberg (control SP) 2012 [30] & RD/Community & 84 & 69 & \multirow{2}{*}{$100 \%$} & \multirow{2}{*}{ Europe } & \multirow{2}{*}{ Linear } & \multirow{2}{*}{ N } & $12(84)$ & \multirow{2}{*}{ W } \\
\hline Dimberg (control ACC) 2012 [30] & RD/AC clinic & 271 & 72 & & & & & $12(271)$ & \\
\hline EAFT 1995 [31] & $\mathrm{RCT}$ & 214 & 71 & $0 \%$ & Europe & - & N & $24(377)$ & $W, A, F$ \\
\hline Ellis 2009 [32] & $\mathrm{RCT}$ & 66 & 68 & $97 \%$ & N. America & Linear & N & $3(17)$ & T \\
\hline Evans 2000 [33] & $\mathrm{PD} / \mathrm{AC}$ clinic & 288 & 76 & $0 \%$ & Europe & NR & N & $24(576)$ & W \\
\hline Evans 2001 [34] & $\mathrm{PD} / \mathrm{AC}$ clinic & 214 & 78 & $N R$ & Europe & $N R$ & $\mathrm{~N}$ & $24(448)$ & W \\
\hline
\end{tabular}


Table 1 Demographics of included studies (Continued)

\begin{tabular}{|c|c|c|c|c|c|c|c|c|c|}
\hline Ezekowitz 2007 [35] & $\mathrm{RCT}$ & 70 & 69 & $100 \%$ & Multinational & $N R$ & $\mathrm{~N}$ & $3(18)$ & W \\
\hline Fang 2004 [36] & RD/AC Clinic & 170 & 78 & 100 & N. America & - & $\mathrm{N}$ & - & W \\
\hline Gallagher 2011 [37] & RD/Community & 18113 & 73 & $72 \%$ & Europe & Linear & $\mathrm{N}$ & 20 (30188) & W \\
\hline Garcia (naïve) 2013 [38] & \multirow{2}{*}{ RCT } & 3888 & 70 & $0 \%$ & \multirow{2}{*}{ Multinational } & \multirow{2}{*}{ Linear } & \multirow{2}{*}{$\mathrm{N}$} & $22(7128)$ & \multirow{2}{*}{ W } \\
\hline Garcia (exp) 2013 [38] & & 5193 & 70 & $100 \%$ & & & & $22(9521)$ & \\
\hline Gladstone 2009 [39] & RD/Community & 423 & 78 & 100 & N. America & - & N & - & W \\
\hline Go 2003 [40] & $\mathrm{RD} / \mathrm{AC}$ clinic & 7445 & 71 & Mixed & N. America & Linear & $\mathrm{N}$ & $21(12958)$ & W \\
\hline Gullov 1998 [41] & $\mathrm{RCT}$ & 170 & 73 & $0 \%$ & Europe & Linear & $\mathrm{N}$ & $25(355)$ & W \\
\hline Gullov 1999 [42] & $\mathrm{RCT}$ & 170 & 73 & 0 & Europe & Linear & $N$ & $25(355)$ & W \\
\hline Gurwitz 1997 [43] & RD/Community & 117 & 83 & $32 \%$ & N. America & Linear & N & $12(117)$ & W \\
\hline Hannon 2011 [44] & PD/Community & 43 & 77 & 100 & Europe & - & N & - & W \\
\hline Hart 2011 [45] & RCT & 523 & NR & 0 & N. America & - & $\mathrm{N}$ & $13(575)$ & W \\
\hline Heidinger 2000 [46] & RD/Community & 753 & 62 & $100 \%$ & Europe & $N R$ & Y & $12(769)$ & NR \\
\hline Hellemons 1999 [47] & $\mathrm{RCT}$ & 131 & 70 & 0 & Europe & - & $\mathrm{N}$ & $32(354)$ & $P, A$ \\
\hline Ho (hypertension) 2011 [48] & \multirow{2}{*}{ RD/Community } & 278 & 70 & \multirow{2}{*}{ NR } & \multirow{2}{*}{ Multinational } & \multirow{2}{*}{ Linear } & \multirow{2}{*}{ N } & $48(1112)$ & \multirow{2}{*}{ W } \\
\hline Ho (no HTN) 2011 [48] & & 198 & 69 & & & & & $48(792)$ & \\
\hline Holmes 2009 [49] & RCT & 244 & 73 & $100 \%$ & Multinational & $N R$ & $\mathrm{~N}$ & $18(366)$ & W \\
\hline Hori 2012 [50] & $\mathrm{RCT}$ & 250 & 71 & $90 \%$ & Asia & Linear & N & $30(625)$ & W \\
\hline Hylek 2003 [51] & RD/Community & 188 & 76 & 100 & N. America & - & $N$ & - & W \\
\hline Hylek 2007 [52] & PD/AC clinic & 472 & 77 & $0 \%$ & N. America & Linear & N & $12(360)$ & W \\
\hline Jacobs 2009 [53] & RD/Community & 90 & NR & $85 \%$ & N. America & Linear & N & $12(90)$ & W \\
\hline Jones 2005 [54] & RD/Community & 2223 & 72 & $100 \%$ & Europe & Linear & $\mathrm{N}$ & $31(5743)$ & W \\
\hline Kalra 2000 [55] & PD/AC clinic & 167 & 77 & $0 \%$ & Europe & Linear & $N$ & $24(296)$ & W \\
\hline Kim 2009 [56] & $\mathrm{RD} / \mathrm{AC}$ clinic & 129 & 64 & $100 \%$ & Asia & Other & N & $24(258)$ & W \\
\hline Kim 2010 [57] & PD/AC clinic & 499 & 73 & $100 \%$ & N. America & Linear & $\mathrm{N}$ & $5(208)$ & W \\
\hline Kulo (warfarin) 2009 [58] & \multirow{2}{*}{ PD/Community } & 60 & 66 & \multirow{2}{*}{$100 \%$} & \multirow{2}{*}{ Europe } & \multirow{2}{*}{$N R$} & \multirow{2}{*}{ N } & $12(60)$ & W \\
\hline Kulo (acenocoumarol) 2009 [58] & & 57 & 68 & & & & & $12(57)$ & A \\
\hline Lee 2012 [59] & RD/Community & 200 & 67 & NR & Asia & Linear & $\mathrm{N}$ & $42(700)$ & W \\
\hline Malik 2000 [60] & $\mathrm{RD} / \mathrm{AC}$ clinic & 247 & 68 & $100 \%$ & N. America & Other & N & $13(268)$ & W \\
\hline Mant 2007 [61] & $\mathrm{RCT}$ & 488 & 82 & $40 \%$ & Europe & Linear & N & $32(1301)$ & W \\
\hline Matchar (control) 2002 [62] & $\mathrm{RCT}$ & 317 & 76 & \multirow{2}{*}{ NR } & \multirow{2}{*}{ N. America } & \multirow{2}{*}{ Linear } & \multirow{2}{*}{$\mathrm{N}$} & $9(238)$ & \multirow{2}{*}{ W } \\
\hline Matchar (prior to ACC) 2002 [62] & PD/AC clinic & 363 & 75 & & & & & $9(272)$ & \\
\hline McBride 2007 [63] & PD/Community & 361 & 71 & $90 \%$ & Europe & Linear & $\mathrm{N}$ & $9(271)$ & W \\
\hline
\end{tabular}


Table 1 Demographics of included studies (Continued)

\begin{tabular}{|c|c|c|c|c|c|c|c|c|c|}
\hline McCormick 2001 [64] & RD/Community & 174 & 87 & $100 \%$ & N. America & Linear & $\mathrm{N}$ & $12(174)$ & W \\
\hline Melamed 2011 [65] & RD/Community & 906 & 72 & $100 \%$ & Israel & Linear & $\mathrm{N}$ & $10(769)$ & W \\
\hline Menzin 2005 [66] & $\mathrm{RD} / \mathrm{AC}$ clinic & 600 & 72 & $63 \%$ & N. America & Linear & $\mathrm{N}$ & $11(525)$ & W \\
\hline Morocutti 1997 [67] & $\mathrm{RCT}$ & 454 & 72 & $N R$ & Europe & - & $\mathrm{N}$ & 12 & W \\
\hline Neree 2006 [68] & RD/Community & 395 & 74 & $100 \%$ & Europe & Linear & N & $4(132)$ & $P, A, W$ \\
\hline Nichol (ACC) 2008 [69] & $\mathrm{RD} / \mathrm{AC}$ clinic & 351 & \multirow{2}{*}{ NR } & \multirow{2}{*}{$100 \%$} & \multirow{2}{*}{ N. America } & \multirow{2}{*}{ Linear } & \multirow{2}{*}{ N } & $31(920)$ & \multirow{2}{*}{ W } \\
\hline Nichol (UC) 2008 [69] & RD/Community & 756 & & & & & & $18(1165)$ & \\
\hline Njaastad 2006 [70] & $\mathrm{RD} / \mathrm{AC}$ clinic & 421 & NR & $0 \%$ & Europe & Linear & $N$ & $14(475)$ & W \\
\hline Nozawa 2001 [71] & PD/Community & 156 & 68 & 100 & Asia & - & $N$ & $22(286)$ & W \\
\hline Ogawa 2011 [72] & RCT & 75 & 72 & 0 & Asia & Linear & N & $3(210)$ & W \\
\hline Okumura $(<70) 2011$ [73] & PD/Community & 208 & NR & $100 \%$ & Asia & Linear & $\mathrm{N}$ & $23(399)$ & W \\
\hline Olsson 2003 [74] & RCT & 1703 & 70 & $73 \%$ & Multinational & NR & $\mathrm{N}$ & $16(2271)$ & W \\
\hline Ono 2005 [75] & PD/Community & 63 & 76 & 0 & Asia & Linear & $\mathrm{N}$ & $28(145)$ & W \\
\hline Patel 2011 [76] & $\mathrm{RCT}$ & 7133 & 73 & $63 \%$ & Multinational & Linear & $\mathrm{N}$ & 20 (11888) & W \\
\hline Pengo 1998 [77] & RCT & 153 & 74 & $0 \%$ & Europe & Linear & N & $14(179)$ & W \\
\hline Pengo 2001 [78] & PD/AC Clinic & 433 & 68 & 0 & Europe & Linear & N & $17(615)$ & W,A \\
\hline Pengo 2010 [79] & RCT & 132 & 79 & $0 \%$ & Multinational & Linear & $\mathrm{N}$ & $64(704)$ & W \\
\hline Perez-Gomez 2004 [80] & RCT & 237 & 70 & $0 \%$ & Europe & $N R$ & $\mathrm{~N}$ & $33(556)$ & A \\
\hline Poli 2007 [81] & PD/AC clinic & 290 & 82 & $100 \%$ & Europe & Linear & $\mathrm{N}$ & $34(814)$ & NR \\
\hline Poli 2009 [82] & PD/AC Clinic & 783 & 75 & $0 \%$ & Europe & Linear & N & $39(2567)$ & W \\
\hline Poli 2009 [83] & PD/AC clinic & 578 & 75 & $100 \%$ & Europe & Linear & N & $38(1854)$ & NR \\
\hline Poli 2009 [84] & PD/AC clinic & 780 & 75 & 100 & Europe & Linear & $\mathrm{N}$ & $36(2406)$ & W \\
\hline Poller (Manual) 2008 [85] & RCT & 2967 & 67 & $0 \%$ & Multinational & Linear & N & $17(4203)$ & W, A, P \\
\hline Sadanaga 2010 [86] & PD/Community & 259 & 74 & 100 & Asia & - & $\mathrm{N}$ & $25(544)$ & W \\
\hline Samsa (ACC) 2000 [87] & RD/AC Clinic & 43 & & & & & & $9(32)$ & \\
\hline Samsa (Rochester) 2000 [87] & RD/Community & 61 & 69 & $N R$ & N. America & Linear & $\mathrm{N}$ & $9(46)$ & W \\
\hline Samsa (R. Triangle) 2000 [87] & RD/Community & 125 & & & & & & $9(94)$ & \\
\hline Schwammenthal 2010 [88] & PD/Community & 111 & 76 & 100 & Israel & - & N & - & W \\
\hline Sconce (vitamin K) 2007 [89] & \multirow{2}{*}{ RCT } & 35 & 75 & \multirow{2}{*}{$100 \%$} & \multirow{2}{*}{ Europe } & \multirow{2}{*}{ Linear } & \multirow{2}{*}{$\mathrm{N}$} & \multirow{2}{*}{$6(18)$} & \multirow{2}{*}{ W } \\
\hline Sconce (placebo) 2007 [89] & & 35 & 74 & & & & & & \\
\hline Shen 2007 [90] & RD/Community & NR & 72 & $55 \%$ & N. America & NR & $\mathrm{N}$ & $40(24179)$ & W \\
\hline Singer 2009 [91] & RD/Community & 9217 & 77 & 100 & N. America & - & $\mathrm{N}$ & 72 (33497) & W \\
\hline SPAF III 1996 [92] & RCT & 523 & 71 & $56 \%$ & N. America & NR & $\mathrm{N}$ & $13(567)$ & W \\
\hline
\end{tabular}


Table 1 Demographics of included studies (Continued)

\begin{tabular}{|c|c|c|c|c|c|c|c|c|c|}
\hline Sullivan (women) 2012 [93] & \multirow{2}{*}{$\mathrm{RCT}$} & 1594 & 71 & $87 \%$ & \multirow{2}{*}{ Multinational } & \multirow{2}{*}{ Linear } & \multirow{2}{*}{ N } & $42(5579)$ & \multirow{2}{*}{ W } \\
\hline Sullivan (men) 2012 [93] & & 2466 & 68 & $86 \%$ & & & & $42(8631)$ & \\
\hline Tincani 2009 [94] & PD/AC Clinic & 90 & 92 & $100 \%$ & Europe & Linear & N & $12(90)$ & W \\
\hline Van Spall 2012 [95] & RCT & 6022 & 72 & $47 \%$ & Multinational & Linear & N & $24(12044)$ & W \\
\hline Voller (self-mgmt) 2005 [96] & \multirow{2}{*}{$\mathrm{RCT}$} & 101 & 65 & \multirow{2}{*}{$0 \%$} & \multirow{2}{*}{ Europe } & \multirow{2}{*}{ NR } & Y & \multirow{2}{*}{$8(68)$} & \multirow{2}{*}{ NP } \\
\hline Voller (UC) 2005 [96] & & 101 & 64 & & & & N & & \\
\hline Walker (MHC) 2011 [97] & \multirow{2}{*}{ RD/AC Clinic } & 22 & \multirow{2}{*}{ NR } & \multirow{2}{*}{$100 \%$} & \multirow{2}{*}{ N. America } & \multirow{2}{*}{ Linear } & \multirow{2}{*}{ N } & $12(22)$ & \multirow{2}{*}{ W } \\
\hline Walker (no MHC) 2011 [97] & & 62 & & & & & & $12(62)$ & \\
\hline Wieloch (AF) 2011 [98] & RD/Community & 11770 & 73 & $100 \%$ & Europe & Linear & N & $12(11770)$ & W \\
\hline Weitz 2010 [99] & RCT & 250 & 66 & $35 \%$ & N. America & Linear & $\mathrm{N}$ & $3(63)$ & W \\
\hline Wyse 2002 [100] & RCT & NR & 70 & NR & N. America & - & N & 42 & W \\
\hline Yamaguchi 2000 [101] & $\mathrm{RCT}$ & 55 & 66 & NR & Asia & - & $\mathrm{N}$ & $22(101)$ & W \\
\hline Yasaka 2001 [102] & PD/Community & 88 & & 100 & Asia & - & N & $22(161)$ & W \\
\hline Yousef 2004 [103] & RD/Community & 739 & 73 & $100 \%$ & Europe & NR & $\mathrm{N}$ & $24(1484)$ & W \\
\hline
\end{tabular}

$\mathrm{VKA}=$ vitamin $\mathrm{K}$ antagonist; $\mathrm{RCT}=$ randomized controlled trial; $\mathrm{RD}=$ retrospective design; $\mathrm{PD}=$ prospective design; $\mathrm{AC}$ clinic $=$ anticoagulation clinic; $\mathrm{AF}=$ atrial fibrillation; $\mathrm{NR}=$ not reported; $\mathrm{N}=\mathrm{North} ; \mathrm{VKA}=\mathrm{Vitamin} \mathrm{K}$ Antagonist; $\mathrm{W}=$ warfarin; $\mathrm{A}=$ acenocoumarol; $\mathrm{T}=$ tecarfarin; $\mathrm{P}=$ phenprocoumon; $\mathrm{F}=$ fenprocoumon 
Table 2 Time in range and percentage of INR values in range and major adverse event rates

\begin{tabular}{|c|c|c|c|c|c|c|c|c|c|c|}
\hline \multirow[t]{2}{*}{ Study, year } & \multirow[t]{2}{*}{$\mathbf{N}$} & \multicolumn{3}{|c|}{ TTR, \% } & \multicolumn{3}{|c|}{ PINRR, \% } & \multicolumn{3}{|c|}{ Event rate, \% per patient-year } \\
\hline & & In range & Below range & Above range & In range & Below range & Above range & Bleeding & $\mathrm{ICH}$ & Ischemic stroke \\
\hline Abdelhafiz 2004 [11] & 402 & 66 & 19.7 & 14.3 & - & - & - & 1.7 & - & - \\
\hline Abdelhafiz (<75) 2008 [12] & 203 & 58 & 16 & 26 & - & - & - & 1.6 & 0 & - \\
\hline Abdelhafiz ( $\geq 75) 2008$ [12] & 199 & 58 & 16 & 26 & - & - & - & 1.9 & 0 & - \\
\hline Albers 2005 [14] & 1962 & 68 & 20 & 12 & - & - & - & 2.8 & 0.2 & 1.1 \\
\hline Anderson 2004 [15] & 87 & - & - & - & 60.4 & 25.2 & 14.3 & - & & \\
\hline Ansell (Italy) 2007 [16] & 177 & 68.9 & 21 & 10.1 & 60 & 26.3 & 13.6 & - & - & - \\
\hline Ansell (Spain) 2007 [16] & 218 & 64.4 & 18.6 & 17 & 59 & 23.3 & 17.7 & - & - & - \\
\hline Ansell (US) 2007 [16] & 686 & 57 & 29.1 & 13.9 & 50.8 & 32 & 17.3 & - & - & - \\
\hline Ansell (Canada) 2007 [16] & 152 & 61 & 25.9 & 13.1 & 58.4 & 27.6 & 14 & - & - & - \\
\hline Ansell (France) 2007 [16] & 278 & 58.1 & 15.4 & 26.5 & 51.3 & 19.7 & 29 & - & - & - \\
\hline Aronow 1999 [17] & 125 & - & - & - & 68 & 26 & 6 & - & - & 11.3 \\
\hline Boulanger 2006 [18] & 6454 & 48 & 38 & 14 & - & - & - & 2.8 & - & - \\
\hline Burton (<75) 2006 [19] & 260 & 68 & 17 & 15 & - & - & - & 2.8 & & \\
\hline Burton (>75) 2006 [19] & 341 & 68 & 19 & 13 & - & - & - & 2.4 & & \\
\hline Cafolla (Manual) 2011 [20] & 576 & 60.4 & - & - & - & - & - & - & - & - \\
\hline Cafolla 2012 [21] & 57 & 64.4 & - & - & - & - & - & 0 & - & - \\
\hline Chung 2011 [23] & 75 & 45.1 & 43.9 & 10.9 & - & - & - & 10.5 & 0 & 0 \\
\hline Connolly 1991 [24] & 187 & 43.7 & 39.6 & 16.6 & - & - & - & 2.1 & 0.4 & 2.1 \\
\hline Connolly 2006 [25] & 3371 & 63.8 & 20.8 & 15.4 & - & - & - & 2.2 & - & 1.0 \\
\hline Currie (stable) 2005 [27] & 787 & 74.9 & - & - & - & - & - & 0.4 & - & - \\
\hline Currie (unstable) 2005 [27] & 726 & 55.7 & - & - & - & - & - & 1.2 & - & - \\
\hline Dimberg (UC) 2012 [30] & 84 & 64.3 & - & - & - & - & - & - & - & - \\
\hline Dimberg (ACC) 2012 [30] & 271 & 73.6 & - & - & - & - & - & - & - & - \\
\hline Ellis 2009 [32] & 66 & 71.4 & - & - & 70.7 & 16.0 & 13.3 & 5.8 & & \\
\hline Evans 2000 [33] & 288 & 55 & 26 & 19 & - & - & - & 3.1 & 0.5 & 5.5 \\
\hline Evans 2001 [34] & 214 & 66 & - & - & - & - & - & 3.1 & 0.7 & 4.2 \\
\hline Ezekowitz 2007 [35] & 70 & 57.2 & - & - & - & - & - & 0 & - & - \\
\hline Gallagher 2011 [37] & 18113 & 63.1 & 20.7 & 16.2 & - & - & - & - & - & - \\
\hline Garcia (naïve) 2013 [38] & 3888 & 61.4 & - & - & - & - & - & 3.0 & 0.8 & - \\
\hline Garcia (exp) 2013 [38] & 5193 & 69.1 & - & - & - & - & - & 3.2 & 0.8 & - \\
\hline Go 2003 [40] & 7445 & 62.5 & 26.8 & 10.7 & - & - & - & 1.5 & 0.4 & 1.1 \\
\hline Gullov 1998 [41] & 170 & 73 & 18 & 9 & - & - & - & 1.1 & 0.6 & 0.8 \\
\hline
\end{tabular}


Table 2 Time in range and percentage of INR values in range and major adverse event rates (Continued)

\begin{tabular}{|c|c|c|c|c|c|c|c|c|c|c|c|}
\hline Gurwitz 1997 [43] & 117 & 39.6 & 44.8 & 15.6 & - & - & - & - & - & - & $\stackrel{\grave{\xi}}{\xi}$ \\
\hline Heidinger 2000 [46] & 753 & - & - & - & 69.5 & 23.5 & 7.0 & 1.7 & - & - & \\
\hline Ho (HTN) 2011 [48] & 278 & 49.7 & 45.4 & 4.9 & - & - & - & 1.7 & 0.5 & 2.2 & \\
\hline Ho (no HTN) 2011 [48] & 198 & 49.8 & 44.2 & 6.0 & - & - & - & & & & \\
\hline Holmes 2009 [49] & 244 & 66 & - & - & - & - & - & 2.7 & - & 1.6 & \\
\hline Hori 2012 [50] & 250 & - & - & - & 51.8 & 43.8 & 4.2 & 0.8 & & 0.3 & \\
\hline Hylek 2007 [52] & 472 & 58 & 29 & 11 & - & - & - & 7.2 & 2.5 & - & \\
\hline Jacobs 2009 [53] & 90 & 49 & 35 & 15 & - & - & - & 5.6 & & & \\
\hline Jones 2005 [54] & 2223 & 68.9 & 16.7 & 15.4 & 53.4 & - & - & - & - & - & \\
\hline Kalra 2000 [55] & 167 & 61 & 26 & 13 & - & - & - & 1.4 & 0.3 & 2.0 & \\
\hline Kim 2009 [56] & 129 & - & - & - & 60.9 & 31.2 & 9.1 & 0 & & & 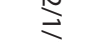 \\
\hline Kim 2010 [57] & 499 & 73.4 & - & - & - & - & - & - & - & - & $\vec{\perp}$ \\
\hline Kulo (warfarin) 2009 [58] & 60 & - & - & - & 51.8 & 42.8 & 5.4 & - & - & - & \\
\hline Kulo (acenocoum) 2009 [58] & 57 & - & - & - & 53.6 & 35.9 & 10.5 & - & - & - & \\
\hline Lee 2012 [59] & 200 & 48.5 & - & - & - & - & - & 6.6 & - & - & \\
\hline Malik (AF) 2000 [60] & 247 & 58.9 & 27.4 & 14.1 & - & - & - & - & - & - & \\
\hline Mant 2007 [61] & 488 & 67 & 19 & 14 & - & - & - & 1.9 & - & 0.8 & \\
\hline Matchar (control) 2002 [62] & 317 & 52.3 & 31.8 & 15.9 & - & - & - & 2.1 & - & - & \\
\hline Matchar (ACC) 2002 [62] & 363 & 55.6 & 31.2 & 13.1 & - & - & - & 2.2 & - & - & \\
\hline McBride 2007 [63] & 361 & 56 & 16 & 28 & - & - & - & - & - & - & \\
\hline McCormick 2001 [64] & 174 & 51 & 36 & 13 & - & - & - & - & - & - & \\
\hline Melamed 2011 [65] & 906 & 48.6 & 32.0 & 19.3 & - & - & - & - & - & - & \\
\hline Menzin 2005 [66] & 600 & 62 & 25 & 13 & - & - & - & 3.6 & - & 1.0 & \\
\hline Neree 2006 [68] & 395 & 53 & - & - & 52.8 & 8.7 & 38.5 & 4.4 & - & - & \\
\hline Nichol (ACC) 2008 [69] & 351 & 68 & 21 & 11 & - & - & - & 2.3 & - & - & \\
\hline Nichol (UC) 2008 [69] & 756 & 42 & 49 & 9 & - & - & - & 6.3 & - & - & \\
\hline Njaastad 2006 [70] & 421 & 71.5 & - & - & - & - & - & 0.8 & - & - & \\
\hline Okumura $(<70) 2011$ [73] & 208 & 46 & 51 & 2 & - & - & - & - & - & - & \\
\hline Olsson 2003 [74] & 1703 & 66 & - & - & - & - & - & 2.2 & - & 1.9 & \\
\hline Patel 2011 [76] & 7133 & 55 & - & - & - & - & - & 3.4 & 0.7 & - & \\
\hline Pengo 1998 [77] & 153 & 70 & 18 & 12 & - & - & - & 2.8 & 0.6 & 0 & قี้ \\
\hline Pengo 2010 [79] & 132 & 65 & 25 & 9 & - & - & - & 3.0 & - & - & \\
\hline Perez-Gomez 2004 [80] & 237 & 65 & 19 & 16 & - & - & - & 1.8 & & & 우 \\
\hline
\end{tabular}


Table 2 Time in range and percentage of INR values in range and major adverse event rates (Continued)

\begin{tabular}{|c|c|c|c|c|c|c|c|c|c|c|}
\hline Poli 2007 [81] & 290 & 69 & 15 & 16 & - & - & - & 2.1 & 1.4 & - \\
\hline Poli 2009 [82] & 783 & 71 & 14 & 15 & - & - & - & 1.4 & 0.7 & 0.9 \\
\hline Poli 2009 [83] & 578 & 68 & 16 & 16 & - & - & - & - & - & - \\
\hline Poller (Manual) 2008 [85] & 2967 & 66.2 & - & - & - & - & - & - & - & - \\
\hline Samsa (ACC) 2000 [87] & 43 & 60.3 & 25.9 & 13.8 & 54.9 & 26.7 & 18.3 & - & - & - \\
\hline Samsa (Rochester) 2000 [87] & 61 & 46.9 & 33.9 & 19.3 & 43.6 & 33.5 & 22.9 & - & - & - \\
\hline Samsa (R. Triangle) 2000 [87] & 125 & 35.6 & 52.1 & 12.2 & 34.2 & 51.0 & 14.8 & - & - & - \\
\hline Sconce (vitamin K) 2007 [89] & 35 & 87 & - & - & - & - & - & - & - & - \\
\hline Sconce (placebo) 2007 [89] & 35 & 78 & - & - & - & - & - & - & - & - \\
\hline Shen 2007 [90] & NR & 54.5 & 36.3 & 9.2 & - & - & - & - & 0.5 & - \\
\hline SPAF III 1996 [92] & 523 & - & - & - & 61 & 25 & 14 & & & \\
\hline Sullivan (women) 2012 [93] & 1594 & 60 & 29 & 11 & - & - & - & - & - & 0.8 \\
\hline Sullivan (men) 2012 [93] & 2466 & 63 & 26 & 14 & - & - & - & - & - & 0.5 \\
\hline Tincani 2009 [94] & 90 & 66 & 19 & 14 & - & - & - & 5.5 & 3.3 & 2.2 \\
\hline Van Spall 2012 [95] & 6022 & 64 & 22 & 13 & - & - & - & - & - & - \\
\hline Voller (self-mgmt) 2005 [96] & 101 & 72.4 & 13.6 & 14 & 67.8 & 15.2 & 17.0 & 2.9 & - & - \\
\hline Voller (UC) 2005 [96] & 101 & 63.6 & 19.5 & 16.9 & 58.5 & 22.1 & 19.4 & 0 & - & - \\
\hline Walker (MHC) 2011 [97] & 22 & 56.8 & 34.2 & 9.0 & - & - & - & - & - & - \\
\hline Walker (no MHC) 2011 [97] & 62 & 65.9 & 30.7 & 3.4 & - & - & - & - & - & - \\
\hline Wieloch (AF) 2011 [98] & 11770 & 76.5 & - & - & - & - & - & 2.6 & - & - \\
\hline Weitz 2010 [99] & 250 & 49.7 & - & - & - & - & - & 1.6 & - & - \\
\hline Yousef 2004 [103] & 739 & - & - & - & 62.8 & 22.9 & 14.3 & 1.9 & - & - \\
\hline
\end{tabular}

$\mathrm{AC}=$ Anticoagulation; $\mathrm{AF}=$ atrial fibrillation; $\mathrm{ICH}=$ Intracranial Hemorrhage; $\mathrm{HTN}=$ Hypertension, INR = international normalized ratio; $\mathrm{MGMT}=$ Management; $\mathrm{N}=\mathrm{Number} ; \mathrm{SP}=\mathrm{Standard}$ Practice; $\mathrm{TR}=$ time in the therapeutic range; PINRR = proportion of INR Measurements in range. 
Table 3 Results of traditional meta-analysis and meta-regression analyses of time in the therapeutic range

\begin{tabular}{|c|c|c|c|c|}
\hline \multirow[b]{2}{*}{ Study-level factors } & \multirow[b]{2}{*}{ No. (\%) } & \multicolumn{3}{|c|}{ Time spent in therapeutic range } \\
\hline & & $\begin{array}{l}\text { Unadjusted pooled mean upon } \\
\text { subgroup analysis, } \%(95 \% \mathrm{CI})\end{array}$ & $\begin{array}{l}\text { Adjusted difference, } \\
\%(95 \% \mathrm{Cl})\end{array}$ & $p$-value \\
\hline All study groups & $78(100)$ & 61 (59 to 62) & NA & NA \\
\hline \multicolumn{5}{|l|}{ Study setting } \\
\hline AC Clinic & $25(32.1)$ & $64(62$ to 66$)$ & $7.2(3.2$ to 11.2$)$ & $<0.001$ \\
\hline $\mathrm{RCT}$ & $26(33.3)$ & 63 (61 to 65$)$ & 9.1 (4.3 to 13.9 ) & $<0.001$ \\
\hline Community & $27(34.6)$ & 55 (52 to 59) & Referent & \\
\hline \multicolumn{5}{|l|}{ Study year } \\
\hline $1990-2000$ & $10(12.8)$ & 53 (46 to 61$)$ & $-4.3(-10.2$ to 1.6$)$ & 0.16 \\
\hline $2001-2007$ & $34(43.6)$ & $64(62$ to 65$)$ & $2.2(-1.7$ to 6.1$)$ & 0.28 \\
\hline 2008-2013 & $34(43.6)$ & 60 (57 to 63) & Referent & \\
\hline \multicolumn{5}{|l|}{ Interpolation method } \\
\hline NR/Other & $11(14.1)$ & 63 (60 to 65) & $-2.1(-6.8$ to 2.6$)$ & 0.38 \\
\hline Linear & $67(85.9)$ & 60 (59 to 62) & Referent & \\
\hline \multicolumn{5}{|l|}{ Self-management } \\
\hline No & $77(98.7)$ & 60 (58 to 62) & $-6.8(-23.7$ to 10.1$)$ & 0.43 \\
\hline Yes & $1(1.3)$ & 72 (68 to 77 ) & Referent & \\
\hline \multicolumn{5}{|l|}{ Geographic region } \\
\hline Europe/UK & $35(44.9)$ & 67 (64 to 69) & 9.7 (6.0 to 13.4$)$ & $<0.001$ \\
\hline Asia & $3(3.8)$ & 47 (44 to 49 ) & $-5.5(-14.7$ to 3.7$)$ & 0.24 \\
\hline Other & $1(1.3)$ & 49 (46 to 52$)$ & $-3.0(-16.1$ to 10.1$)$ & 0.65 \\
\hline Multinational & $13(16.7)$ & 61 (57 to 65) & $1.8(-4.1$ to 7.7$)$ & 0.55 \\
\hline North America & $26(33.3)$ & 55 (50 to 60$)$ & Referent & \\
\hline \multicolumn{5}{|l|}{ VKA experience } \\
\hline NR/Mixed & $21(26.9)$ & 54 (49 to 60$)$ & $-4.6(-8.3$ to -0.9$)$ & 0.02 \\
\hline No & $17(21.8)$ & 63 (60 to 66) & $-5.3(-9.6$ to -1.0$)$ & 0.02 \\
\hline Yes & $40(51.3)$ & 63 (60 to 66$)$ & Referent & \\
\hline \multicolumn{5}{|c|}{ Duration of VKA treatment } \\
\hline$\geq 1$ year & $59(75.6)$ & 62 (60 to 63) & $2.7(-1.7$ to 7.1$)$ & 0.23 \\
\hline$<1$ year & $19(24.4)$ & 58 (52 to 65$)$ & Referent & \\
\hline
\end{tabular}

$\mathrm{AC}=$ anticoagulation; $\mathrm{Cl}=$ confidence interval; $\mathrm{NA}=$ not applicable; $\mathrm{No} .=$ number of study arms; $\mathrm{NR}=$ Not Reported; $\mathrm{RCT}=$ randomized controlled trial; $\mathrm{UK}=\mathrm{United}$ Kingdom, VKA = Vitamin $\mathrm{K}$ Antagonists.

29-63\%) occurred at INRs $<2.0$ and $>3.0$, respectively, but a high degree of heterogeneity was still present $\left(\mathrm{I}^{2}=76 \%\right.$ and $85 \%$, respectively) (Figures 4 and 5). Furthermore, when studies that allowed previously reported INRs (more than 48 hours prior to an event) were excluded, we found a higher proportion of events that occurred outside the therapeutic range for $59 \%$ of thromboembolic events $(95 \%$ CI, $51-66 \%)$ and $47 \%$ of hemorrhagic events $(95 \% \mathrm{CI}$, $37-58 \%)$.

\section{Discussion}

In a pooled analysis of AF studies performed worldwide between 1990 and June 2013, we found patients spent only $61 \%$ of their TTR and only $56 \%$ of their measured
INRs were in range. Moreover, when patients were out of range, they were more likely to be below range and at an increased risk of thrombosis, as compared to being above range with an increased risk of bleeding. While, thromboembolic and hemorrhagic events did occur in patients with a therapeutic INR; we demonstrated more than half of all thromboembolic events occurred when the INR was less than 2.0 and more than $40 \%$ of all hemorrhagic events happened at an INR $>3.0$. As such, the efficacy and safety of VKAs appear strongly tethered to the quality of INR control achieved.

Our meta-regression analyses showed three important findings. First, patients having their VKAs managed in the community setting spent significantly less TTR when 
Table 4 Results of traditional meta-analysis and meta-regression analyses of proportion of INR measurements in range

\begin{tabular}{|c|c|c|c|c|}
\hline \multirow[b]{2}{*}{ Study-level factors } & \multirow[b]{2}{*}{ No. (\%) } & \multicolumn{3}{|c|}{ Proportion of INR measurements in range } \\
\hline & & $\begin{array}{l}\text { Unadjusted pooled mean upon } \\
\text { subgroup analysis, } \%(95 \% \mathrm{Cl})\end{array}$ & $\begin{array}{c}\text { Adjusted difference, } \\
\%(95 \% \mathrm{Cl})\end{array}$ & $p$-value \\
\hline All study groups & $24(100)$ & 56 (53 to 59$)$ & NA & NA \\
\hline \multicolumn{5}{|l|}{ Study setting } \\
\hline AC Clinic & $5(20.8)$ & 60 (58 to 62) & $6.9(-0.02$ to 15.2$)$ & 0.13 \\
\hline $\mathrm{RCT}$ & $5(20.8)$ & 61 (54 to 68) & $5.6(-0.06$ to 17.0$)$ & 0.36 \\
\hline Community & $14(58.3)$ & 54 (50 to 57$)$ & Referent & \\
\hline \multicolumn{5}{|l|}{ Study year } \\
\hline $1990-2000$ & $6(25.0)$ & 54 (46 to 64$)$ & $3.3(-25.3$ to 31.9$)$ & 0.82 \\
\hline $2001-2007$ & $13(54.2)$ & 56 (54 to 59) & $-2.8(-14.8$ to 9.2$)$ & 0.65 \\
\hline $2008-2013$ & $5(20.8)$ & 56 (51 to 62$)$ & Referent & \\
\hline \multicolumn{5}{|l|}{ Self-management } \\
\hline No & $22(91.7)$ & 55 (43 to 57$)$ & $-9.3(-32.4$ to 13.8$)$ & 0.45 \\
\hline Yes & $2(8.3)$ & 69 (67 to 72) & Referent & \\
\hline \multicolumn{5}{|l|}{ Geographic region } \\
\hline Europe/UK & $13(54.2)$ & 57 (54 to 60) & $-1.7(-10.7$ to 7.3$)$ & 0.71 \\
\hline Asia & $2(8.3)$ & 56 (48 to 66) & $-8.8(-26.4$ to 8.8$)$ & 0.32 \\
\hline Other & $0(0)$ & NA & NA & NA \\
\hline Multinational & $0(0)$ & NA & NA & NA \\
\hline North America & $9(37.5)$ & 54 (48 to 62$)$ & Referent & \\
\hline \multicolumn{5}{|l|}{ VKA experience } \\
\hline NR/Mixed & $5(20.8)$ & 51 (41 to 63) & $-5.7(-34.7$ to 23.3$)$ & 0.83 \\
\hline No & $3(12.5)$ & 63 (55 to 73) & $7.9(-15.2$ to 31.0$)$ & 0.65 \\
\hline Yes & $17(70.8)$ & 56 (54 to 59$)$ & Referent & \\
\hline \multicolumn{5}{|c|}{ Duration of VKA treatment } \\
\hline$\geq 1$ year & $17(70.8)$ & 57 (54 to 60) & $9.1(-0.5$ to 18.7$)$ & 0.09 \\
\hline$<1$ year & $7(29.2)$ & 53 (44 to 65$)$ & Referent & \\
\hline
\end{tabular}

$\mathrm{AC}=$ anticoagulation; $\mathrm{Cl}=$ confidence interval; $\mathrm{NA}=$ not applicable; No. = number of study arms; $\mathrm{NR}=$ not reported; $\mathrm{RCT}=$ randomized controlled trial; $\mathrm{UK}=\mathrm{United}$ Kingdom; VKA = Vitamin K Antagonists.

compared to patients managed in an anticoagulation clinic or in a RCT setting. This data supports the growing body of literature demonstrating that patients whose care is managed in an anticoagulation clinic have better outcomes (improved TTR, lower rates of major bleeding and thrombosis, and decreased health care costs) than those managed in community practice [104-106]. The improved INR control in RCTs and anticoagulation clinics may be the result of increasing the frequency of monitoring, providing more organized care and focusing more on improving poor VKA-drug persistence (as low as 68\% at 6-months) $[107,108]$ than typically done in community settings. Unfortunately, not all patients receiving a VKA have access to anticoagulation clinics [109]. In fact, it is estimated that only about one-third of patients receiving a VKA in the US have access to an anticoagulation clinic due to time, distance, economic or other access-to-care issues. Second, patients who were VKA-naive spent significantly less time in the therapeutic range than VKA-experienced patients. This is likely a result of poorer INR control early on in treatment, as clinicians attempt to control the INR within the narrow therapeutic window without any prior knowledge of the patient's-specific dosing requirements. It may also suggest that patients learn to manage their VKA better over time. This observation may warrant researchers to stratify analyses according to whether patients are newly initiated to warfarin or experienced warfarin patients. Finally, people in North America spent significantly less time in the therapeutic range than those in Europe or the UK. This may be a result of North America's near exclusive use of warfarin, which has been shown in previous analyses to result in as much as a 9\% lower TTR compared to other VKAs [6], as well as, North America's less wide-spread use of proven strategies to increase 


\begin{tabular}{|c|c|c|c|c|c|}
\hline Study & $\begin{array}{c}\text { No. of } \\
\text { Thromboembolic }\end{array}$ & Relative Weight, \% & \multicolumn{3}{|c|}{ Proportion ( $95 \%$ Confidence Interval) } \\
\hline Akdeniz 2005 & $4 / 4$ & 1.751003 & $1.00(0.40-1.00)$ & & $\rightarrow$ \\
\hline Albers 2005 & $9 / 23$ & 4.072459 & $0.39(0.20-0.61)$ & 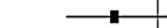 & \\
\hline Cheung 2005 & $28 / 35$ & 4.608403 & $0.80(0.63-0.92)$ & & $\longrightarrow$ \\
\hline Connolly 1991 & $4 / 5$ & 1.989794 & $0.80(0.29-0.99)$ & & \\
\hline Copland 2001 & $0 / 2$ & 1.183084 & $0.00(0.00-0.84)$ & & \\
\hline Dentali 2012 & $3 / 3$ & 1.483885 & $1.00(0.29-1.00)$ & & \\
\hline EAFT 1995 & $7 / 21$ & 3.947262 & $0.33(0.15-0.57)$ & & \\
\hline Gladstone 2009 & $299 / 423$ & 6.07053 & $0.71(0.66-0.75)$ & & $\rightarrow$ \\
\hline Go 2003 & $87 / 137$ & 5.72147 & $0.64(0.55-0.72)$ & & \\
\hline Gullov 1998 & $0 / 6$ & 2.204538 & $0.00(0.00-0.46)$ & & \\
\hline Hannon 2011 & $13 / 27$ & 4.286085 & $0.48(0.29-0.68)$ & & - \\
\hline Holmes 2009 & $6 / 6$ & 2.204538 & $1.00(0.54-1.00)$ & & \\
\hline Hylek 2003 & $117 / 188$ & 5.856179 & $0.62(0.55-0.69)$ & & 1 \\
\hline Kalra 2000 & $4 / 6$ & 2.204538 & $0.67(0.22-0.96)$ & & \\
\hline Morocutti 1997 & $2 / 10$ & 2.883512 & $0.20(0.03-0.56)$ & & \\
\hline Ogawa 2011 & $0 / 2$ & 1.183084 & $0.00(0.00-0.84)$ & & \\
\hline Olsson 2003 & $17 / 67$ & 5.259898 & $0.25(0.16-0.37)$ & $\rightarrow-$ & \\
\hline Ono 2005 & $3 / 3$ & 1.483885 & $1.00(0.29-1.00)$ & & \\
\hline Pengo 1998 & $2 / 2$ & 1.183084 & $1.00(0.16-1.00)$ & & \\
\hline Pengo 2001 & $4 / 10$ & 2.883512 & $0.40(0.12-0.74)$ & & \\
\hline Poli 2009 & $20 / 40$ & 4.761229 & $0.50(0.34-0.66)$ & $\longrightarrow$ & \\
\hline Poli 2009 & $13 / 31$ & 4.461614 & $0.42(0.25-0.61)$ & 1 & \\
\hline Sadanaga 2010 & $9 / 10$ & 2.883512 & $0.90(0.55-1.00)$ & & $\rightarrow$ \\
\hline Schwammenthal 2010 & $63 / 111$ & 5.610444 & $0.57(0.47-0.66)$ & - & - \\
\hline Singer 2009 & $249 / 396$ & 6.058401 & $0.63(0.58-0.68)$ & & $-\pi$ \\
\hline Tincani 2009 & $1 / 1$ & 0.841799 & $1.00(0.03-1.00)$ & & \\
\hline Wyse 2002 & $44 / 88$ & 5.464982 & $0.50(0.39-0.61)$ & $\rightarrow$ & \\
\hline Yamaguchi 2000 & $0 / 1$ & 0.841799 & $0.00(0.00-0.98)$ & & \\
\hline Yasaka 2001 & $4 / 4$ & 1.751003 & $1.00(0.40-1.00)$ & & \\
\hline Yousef 2004 & $27 / 44$ & 4.864473 & $0.61(0.45-0.76)$ & & $=$ \\
\hline Overall Estimate & & & $0.57(0.50-0.64)$ & $\frac{\theta}{10}$ & $t$ \\
\hline$I^{2}=80 \%$ & & & & 0.00 & 0.75 \\
\hline
\end{tabular}

Figure 2 Results of random-effects meta-analysis of the proportion of thromboembolic events that occurred when INRs were below 2.0. The squares represent individual studies, and the size of the square represents the weight given to each study in the meta-analysis. Error bars represent 95\% confidence intervals. The diamond represents the combined results. List of studies shows name of first author and year of publication. $\mathrm{Cl}=$ Confidence Interval.

TTR such as and anticoagulation clinics as noted above $[6,8,9,109]$ and patient self-monitoring [6].

Our systematic review and meta-analysis supports and extends the knowledge of VKA use in contemporary practice. Our TTR or PINRR results are in general agreement with the systematic reviews by van Walraven et al. [6] and Cios et al. [9], but these analyses included all therapeutic indications for VKAs, which lowers their applicability for an AF-specific population. The meta-analyses by Baker et al. [8] and Wan et al. [7] focused on AF only populations, but each had some limitations. The Baker metaanalysis was limited to a United States population, and therefore, contained only 8 studies. Despite this, they did find that care within an anticoagulation clinic yielded a higher TTR than usual care in the community, a finding we extend into the worldwide AF population as well. The Wan meta-analysis was published in 2008, evaluated a worldwide AF population and found similar TTR and PINRR results to our own. However, they only included 47 VKA groups from 38 published studies. They found that TTR was significantly correlated with PINRR in retrospective studies and TTR was significantly negatively correlated to both major hemorrhage and thromboembolic events. Given the intense focus on thromboembolism in contemporary practice, our literature search was able to almost double the number of articles included in the Wan et al. by updating the search to 2013. Over the 5 years since the publication of Wan et al.'s metaanalysis, we found the TTR and PINRR to be only slightly increased (overall TTR increased from 57\% to $61 \%$ and PINRR from 51 to 56\%) [7]. This suggests that we still have a long way to go to enhance the quality of INR control. Our findings that the majority of thromboembolic events happen in AF patients when the INR is less than 2.0 and more than $40 \%$ of hemorrhages occur when an INR is greater than 3.0 confirms the findings from the systematic review of Oake et al. [10]. Their systematic review did not limit the studies to an AF population and was published in 2009 , so our findings through June 2013 in the target AF population extend knowledge in this area as well.

There are several limitations of our meta-analysis worth discussion. First, the potential for publication bias and possibility of missed eligible articles could exist. However, we consider this risk to be minimized due to our systematic search strategy and manual backwards citation tracking. In addition, having such a large cadre of studies, as we do in our meta-analysis, minimizes the impact that a 


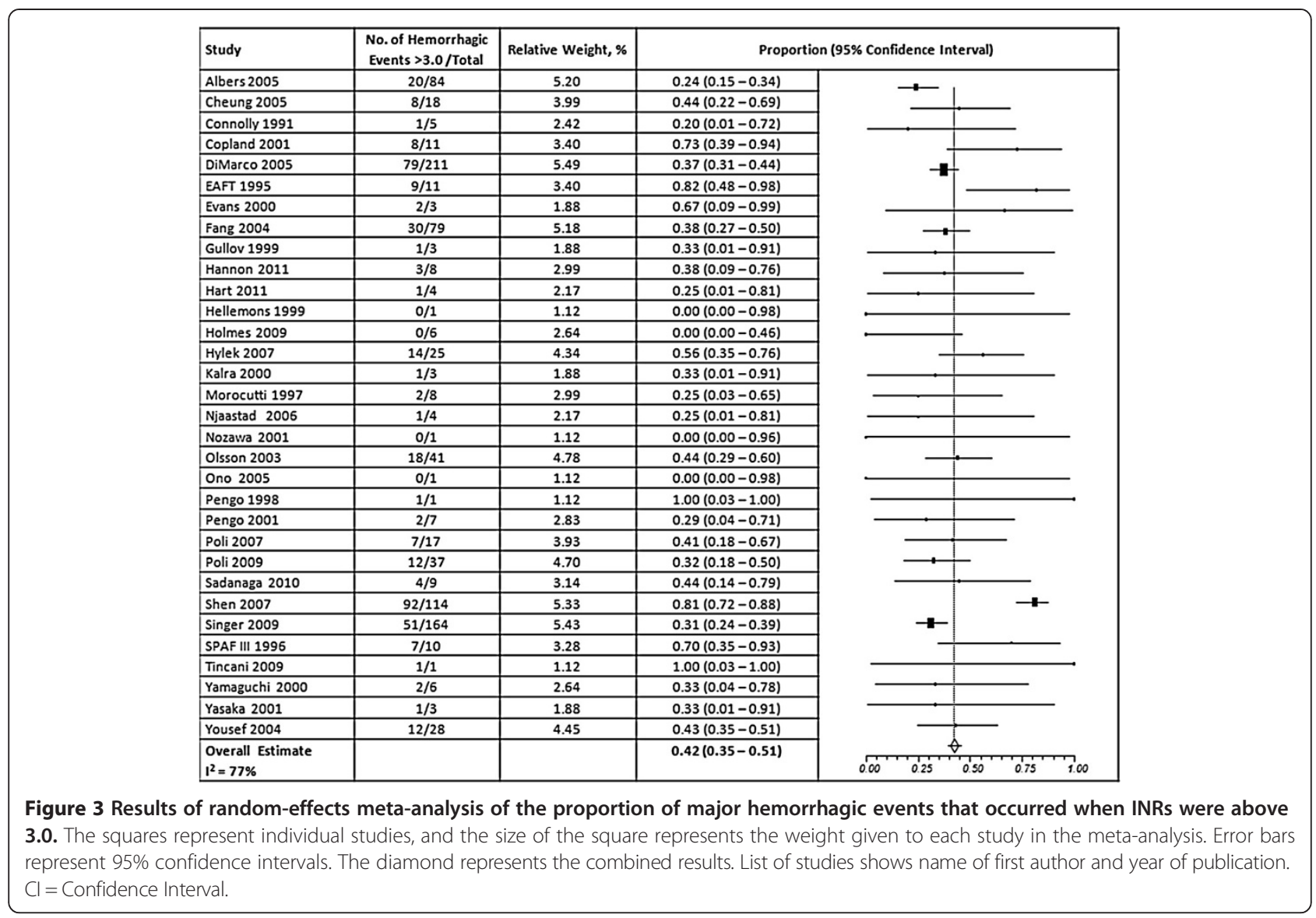

\begin{tabular}{|l|c|c|c|c|}
\hline Study & $\begin{array}{c}\text { No. of Ischemic } \\
\text { Strokes }<2.0 / \text { Total }\end{array}$ & Relative Weight, \% & \multicolumn{2}{|c|}{ Proportion (95\% Confidence Interval) } \\
\hline Akdeniz 2005 & $4 / 4$ & 2.56 & $1.00(0.40-1.00)$ \\
\hline Albers 2005 & $9 / 23$ & 6.23 & $0.39(0.20-0.61)$ \\
\hline Coppland 2001 & $0 / 2$ & 1.71 & $0.00(0.00-0.84)$ \\
\hline Gladstone 2009 & $299 / 423$ & 9.65 & $0.71(0.66-0.75)$ \\
\hline Go 2003 & $87 / 137$ & 9.03 & $0.64(0.55-0.72)$ \\
\hline Gullov 1998 & $0 / 6$ & 3.25 & $0.00(0.00-0.46)$ \\
\hline Hannon 2011 & $13 / 27$ & 6.58 & $0.48(0.29-0.68)$ \\
\hline Holmes 2009 & $6 / 6$ & 3.25 & $1.00(0.54-1.00)$ \\
\hline Hylek 2003 & $117 / 188$ & 9.27 & $0.62(0.55-0.69)$ \\
\hline Kalra 2000 & $4 / 6$ & 3.25 & $0.67(0.22-0.96)$ \\
\hline Morocutti 1997 & $2 / 10$ & 4.31 & $0.20(0.03-0.56)$ \\
\hline Ogawa 2011 & $0 / 2$ & 1.71 & $0.00(0.00-0.84)$ \\
\hline Ono 2005 & $2 / 2$ & 1.71 & $1.00(0.16-1.00)$ \\
\hline Pengo 2001 & $2 / 2$ & 1.71 & $1.00(0.16-1.00)$ \\
\hline Poli 2009 & $13 / 31$ & 6.87 & $0.42(0.25-0.61)$ \\
\hline Schwammenthal 2010 & $63 / 111$ & 8.84 & $0.57(0.47-0.66)$ \\
\hline Tincani 2009 & $1 / 1$ & 1.21 & $1.00(0.29-1.00)$ \\
\hline Wyse 2002 & $44 / 88$ & 8.58 & $0.58(0.39-0.75)$ \\
\hline Yamaguchi 2000 & $0 / 1$ & 1.21 & $0.00(0.00-0.98)$ \\
\hline Yasaka 2001 & $3 / 3$ & 2.16 & $1.00(0.29-1.00)$ \\
\hline Yousef 2004 & $18 / 31$ & 6.87 & $0.58(0.39-0.75)$ \\
\hline Overall Estimate & & & $0.56(0.48-0.64)$ \\
\hline 12=76\% & & & \\
\hline
\end{tabular}

Figure 4 Results of random-effects meta-analysis of the proportion of ischemic strokes that occurred when INRs were below 2.0. The squares represent individual studies, and the size of the square represents the weight given to each study in the meta-analysis. Error bars represent $95 \%$ confidence intervals. The diamond represents the combined results. List of studies shows name of first author and year of publication. $\mathrm{Cl}=$ Confidence Interval. 


\begin{tabular}{|l|c|c|c|c|}
\hline Study & $\begin{array}{c}\text { No. of Intracranial Hemorrhages } \\
>3.0 / \text { Total }\end{array}$ & Relative Weight, \% & \multicolumn{2}{|c|}{ Proportion (95\% Confidence Interval) } \\
\hline Connolly 1991 & $1 / 1$ & 4.031717 & $1.00(0.03-1.00)$ \\
\hline Copland 2001 & $2 / 3$ & 5.872573 & $0.67(0.09-0.99)$ \\
\hline Evans 2000 & $2 / 3$ & 5.872573 & $0.67(0.09-0.99)$ \\
\hline Fang 2004 & $30 / 79$ & 10.371242 & $0.38(0.27-0.50)$ \\
\hline Gullov 1999 & $1 / 2$ & 5.096844 & $0.50(0.01-0.99)$ \\
\hline Hannon 2011 & $3 / 8$ & 7.868527 & $0.38(0.09-0.76)$ \\
\hline Hellemons 1999 & $0 / 1$ & 4.031717 & $0.00(0.00-0.98)$ \\
\hline Holmes 2009 & $0 / 6$ & 7.301315 & $0.00(0.00-0.46)$ \\
\hline Kalra 2000 & $1 / 1$ & 4.031717 & $1.00(0.03-1.00)$ \\
\hline Morocutti 1997 & $0 / 4$ & 6.462742 & $0.00(0.00-0.60)$ \\
\hline Pengo 1998 & $1 / 1$ & 4.031717 & $1.00(0.03-1.00)$ \\
\hline Shen 2007 & $92 / 114$ & 10.500086 & $0.81(0.72-0.88)$ \\
\hline Singer 2009 & $51 / 194$ & 10.591222 & $0.31(0.24-0.39)$ \\
\hline Tincani 2009 & $1 / 1$ & 4.031717 & $1.00(0.03-1.00)$ \\
\hline Yamaguchi 2000 & $0 / 1$ & 4.031717 & $0.00(0.00-0.98)$ \\
\hline Yasaka 2001 & $1 / 3$ & 5.872573 & $0.33(0.01-0.91)$ \\
\hline $\begin{array}{l}\text { Overall Estimate } \\
\mathbf{2}^{2}=85 \%\end{array}$ & \multicolumn{3}{|}{} & \\
\hline
\end{tabular}

Figure 5 Results of Random-Effects Meta-Analysis of the Proportion of Intracranial Hemorrhages That Occurred When INRs Were

Above 3.0. The squares represent individual studies, and the size of the square represents the weight given to each study in the meta-analysis. Error bars represent 95\% confidence intervals. The diamond represents the combined results. List of studies shows name of first author and year of publication. $\mathrm{Cl}=$ Confidence Interval; $\mathrm{ICH}=$ Intracranial hemorrhage.

missed individual study might have on our pooled result. Another limitation of our analysis includes the fact that very few identified studies evaluated PINRR. As a result, our meta-regression analysis was likely underpowered; although similar trends could be seen to TTR. Future evaluation of PINRR should be conducted when there is a sufficient literature base to make firmer conclusions. Another limitation of our analysis is the possibility of a language bias; as we only included English language studies which may not represent all of the published evidence. Finally, there was a high degree of statistical heterogeneity observed in our analyses, suggesting that the included studies varied clinically and/or methodologically. However, this was one of our rationales for conducting meta-regression. In our meta-regression analyses, we found some potential explanations for the identified heterogeneity; although there may still be additional factors that we could not think to evaluate or for which there is insufficient data to allow evaluation.

\section{Conclusions}

Patients on VKAs for thromboembolism prevention in AF are frequently outside the normal INR range and tend to be under-anticoagulated rather than over-anticoagulated. While, thromboembolic and hemorrhagic events do occur patients with a therapeutic INR; patients with an INR $<2.0$ make up many of the cases of thromboembolism, while those with an INR $>3.0$ make up many of the cases of major hemorrhage. Managing anticoagulation outside of a clinical trial or anticoagulation clinic is associated with poorer INR control as is initiation of therapy in patients who are VKA-naïve. Patients in Europe/UK have better INR control than those in North America.

\section{Additional file}

Additional file 1: Medline search strategy and forest plots for time and percent of INRs in, below and above range.

\section{Abbreviations}

AF: Atrial fibrillation; VKA: Vitamin K antagonist; TTR: Time in therapeutic range; PINRR: Proportion of INR measurements in range; INR: International normalized ratio; Cl: Confidence interval; UK: United Kingdom; RCT: Randomized controlled trial; ICH: Intracranial hemorrhage.

\section{Competing interests}

Dr. Coleman has received honoraria for participation on advisory boards and Speaker's bureaus and has received research funding from Janssen Scientific Affairs. Drs Raut and Schein are paid employees of Janssen Pharmaceuticals. Drs. Mearns, White and Kohn and Mr./Ms. Hawthorne, Song and Meng have no conflicts to report.

\section{Authors' contributions}

Study concept and design: ESM, MKR, JRS, CIC. Acquisition of data: ESM, CGK, JH, JSS, JM. Analysis and interpretation of data: ESM, CMW, CGK, JH, JSS, JM, MKR, JRS, CIC. Drafting of the manuscript: ESM, CMW, CGK, CIC. Critical revision of the manuscript for important intellectual content: ESM, CMW, MKR, JRS, CIC. Administrative, technical, or material support: ESM, MKR, JRS, CIC. Study supervision: ESM, CMW, CIC. ESM and CIC had full access to all the data in the study and take responsibility for the integrity of the data and the accuracy of the data analysis. All authors read and approved the final manuscript.

\section{Acknowledgements}

This study was funded by Janssen Scientific Affairs, LLC. The authors maintained full control over the design and conduct of the study; collection, management, analysis, and interpretation of the data; and preparation and review of the manuscript. Janssen reviewed the final manuscript prior to submission.

\section{Author details}

'Department of Pharmacy Practice, University of Connecticut School of Pharmacy, 69 N Eagleville Road, Storrs, CT 06269-3092, USA. ${ }^{2}$ Hartford Hospital Division of Cardiology, 80 Seymour Street, Hartford, CT 06102-5037, USA. ${ }^{3}$ Janssen Scientific Affairs, LLC, Raritan, NJ, USA. 
Received: 28 February 2014 Accepted: 6 May 2014

Published: 24 June 2014

\section{References}

1. Wolf PA, Abbott RD, Kannel WB: Atrial fibrillation: a major contributor to stroke in the elderly: the Framingham Heart Study. Arch Intern Med 1987, 147(9):1561-1564.

2. Atrial Fibrillation Investigators: Risk factors for stroke and efficacy of antithrombotic therapy in atrial fibrillation. Analysis of pooled data from five randomized controlled trials. Arch Intern Med 1994, 154(13):1449-1457.

3. Fuster V, Rydén LE, Cannom DS, Crijns HJ, Curtis AB, Ellenbogen KA, Halperin JL, Le Heuzey JY, Kay GN, Lowe JE, Olsson SB, Prystowsky EN, Tamargo JL, Wann S, Smith SC Jr, Jacobs AK, Adams CD, Anderson JL, Antman EM, Halperin JL, Hunt SA, Nishimura R, Ornato JP, Page RL, Riegel B, Priori SG, Blanc JJ, Budaj A, Camm AJ, Dean V, Deckers JW, Despres C, Dickstein K, Lekakis J, McGregor K, Metra M, Morais J, Osterspey A, Tamargo JL, Zamorano JL: ACC/AHA/ESC 2006 guidelines for the management of patients with atrial fibrillation. Circulation 2006, 114(7):e257-e354

4. Singer DE, Albers GW, Dalen JE, Fang MC, Go AS, Halperin JL, Lip GY, Manning WJ, American College of Chest Physicians: Antithrombotic therapy in atrial fibrillation: American college of chest physicians evidence-based clinical practice guidelines (8th Edition). Chest 2008, 133(6 Suppl):546S-592S.

5. Ansell J, Hirsh J, Hylek E, Jacobson A, Crowther M, Palareti G, American College of Chest Physicians: Pharmacology and management of the vitamin $\mathrm{K}$ antagonists: American college of chest physicians evidence-based clinical practice guidelines (8th Edition). Chest 2008, 133 (6 Suppl):160S-198S.

6. van Walraven C, Jennings A, Oake N, Fergusson D, Forster AJ: Effect of study setting on anticoagulation control: a systematic review and metaregression. Chest 2006, 129(5):1155-1166

7. Wan Y, Heneghan C, Perera R, Roberts N, Hollowell J, Glasziou P, Bankhead C, $\mathrm{X} \cup \mathrm{Y}$ : Anticoagulation control and prediction of adverse events in patients with atrial fibrillation: a systematic review. Circ Cardiovasc Qual Outcomes 2008, 1(2):84-91.

8. Baker WL, Cios DA, Sander SD, Coleman Cl: Meta-analysis to assess the quality of warfarin control in atrial fibrillation patients in the United States. J Manag Care Pharm 2009, 15(3):244-252.

9. Cios DA, Baker WL, Sander SD, Phung OJ, Coleman Cl: Evaluating the impact of study-level factors on warfarin control in U.S.-based primary studies: a meta-analysis. Am J Health Syst Pharm 2009, 66(10):916-925.

10. Oake N, Fergusson DA, Forster AJ, van Walraven C: Frequency of adverse events in patients with poor anticoagulation: a meta-analysis. CMAJ 2007 176(11):1589-1594.

11. Abdelhafiz AH, Wheeldon NM: Results of an open-labeled, prospective study of anticoagulant therapy for atrial fibrillation in an outpatient anticoagulation clinic. Clin Ther 2004, 26(9):1470-1478.

12. Abdelhafiz $\mathrm{AH}$, Wheeldon NM: Risk factors for bleeding during anticoagulation of atrial fibrillation in older and younger patients in clinical practice. Am J Geriatr Pharmacother 2008, 6(1):1-11.

13. Akdeniz B, Türker S, Oztürk V, Badak O, Okan T, Aslan O, Kozan O, Kirimli O, Aytekin D, Bariş N, Güneri S: Cardioversion under the guidance of transesophageal echochardiograhy in persistent atrial fibrillation: results with low molecular weight heparin. Int J Cardiol 2005, 98(1):49-55.

14. Albers GW, Diener HC, Frison L, Grind M, Nevinson M, Partridge S, Halperin JL, Horrow J, Olsson SB, Petersen P, Vahanian A, SPORTIF Executive Steering Committee for the SPORTIF V Investigators: Ximelagatran vs warfarin for stroke prevention in patients with nonvalvular atrial fibrillation: a randomized trial. JAMA 2005, 293(6):690-698.

15. Anderson RJ: Cost analysis of a managed care decentralized outpatient pharmacy anticoagulation service. J Manag Care Pharm 2004, 10(2):159-165.

16. Ansell J, Hollowell J, Pengo V, Martinez-Brotons F, Caro J, Drouet L: Descriptive analysis of the process and quality of oral anticoagulation management in real-life practice in patients with chronic non-valvular atrial fibrillation: the international study of anticoagulation management (ISAM). J Thromb Thrombolysis 2007, 23(2):83-91.

17. Aronow WS, Ahn C, Kronzon I, Gutstein H: Incidence of new thromboembolic stroke in persons 62 years and older with chronic atrial fibrillation treated with warfarin versus aspirin. J Am Geriatr Soc 1990, 47(3):366-368

18. Boulanger L, Kim J, Friedman M, Hauch O, Foster T, Menzin J: Patterns of use of antithrombotic therapy and quality of anticoagulation among patients with non-valvular atrial fibrillation in clinical practice. Int J Clin Pract 2006, 60(3):258-264.

19. Burton C, Isles C, Norrie J, Hanson R, Grubb E: The safety and adequacy of antithrombotic therapy for atrial fibrillation: a regional cohort study. Br J Gen Pract 2006, 56(530):697-702.

20. Cafolla A, Melizzi R, Baldacci E, Pignoloni P, Dragoni F, Campanelli M, Caraccini R, Foà R: "Zeus" a new oral anticoagulant therapy dosing algorithm: a cohort study. Thromb Res 2011, 128(4):325-330.

21. Cafolla A, Campanelli M, Baldacci E, Potasso L, Bochicchio R, Dragoni F, Foà R: Oral anticoagulant therapy in Italian patients $80 \mathrm{yr}$ of age or older with atrial fibrillation: a pilot study of low vs. standard PT/INR targets. Eur J Haematol 2012, 89(1):81-86.

22. Cheung CM, Tsoi TH, Huang CY: The lowest effective intensity of prophylactic anticoagulation for patients with atrial fibrillation. Cerebrovasc Dis 2005, 20(2):114-119.

23. Chung N, Jeon HK, Lien LM, Lai WT, Tse HF, Chung WS, Lee TH, Chen SA Safety of edoxaban, an oral factor Xa inhibitor, in Asian patients with non-valvular atrial fibrillation. Thromb Haemost 2011, 105(3):535-544.

24. Connolly SJ, Laupacis A, Gent M, Roberts RS, Cairns JA, Joyner C: Canadian atrial fibrillation anticoagulation (CAFA) study. J Am Coll Cardiol 1991, 18(2):349-355

25. ACTIVE Writing Group of the ACTIVE Investigators, Connolly S, Pogue J, Hart R, Pfeffer M, Hohnloser S, Chrolavicius S, Pfeffer M, Hohnloser S, Yusuf S: Clopidogrel plus aspirin versus oral anticoagulation for atrial fibrillation in the Atrial fibrillation Clopidogrel Trial with Irbesartan for prevention of Vascular Events (ACTIVE W): a randomised controlled trial. Lancet 2006, 367(9526):1903-1912.

26. Copland M, Walker ID, Tait RC: Oral anticoagulation and hemorrhagic complications in an elderly population with atrial fibrillation. Arch Intern Med 2001, 161(17):2125-2128.

27. Currie CJ, McEwan P, Emmas C, Morgan CL, Peters JR: Anticoagulation in patients with non-valvular atrial fibrillation: an evaluation of stability and early factors that predict longer-term stability on warfarin in a large UK population. Curr Med Res Opin 2005, 21(12):1905-1913.

28. Dentali F, Pignatelli P, Malato A, Poli D, Di Minno MN, Di Gennaro L, Rancan E, Pastori D, Grifoni E, Squizzato A, Siragusa S, Di Minno G, Ageno W: Incidence of thromboembolic complications in patients with atrial fibrillation or mechanical heart valves with a subtherapeutic international normalized ratio: a prospective multicenter cohort study. Am J Hematol 2012, 87(4):384-387

29. DiMarco JP, Flaker G, Waldo AL, Corley SD, Greene HL, Safford RE, Rosenfeld LE, Mitrani G, Nemeth M, AFFIRM Investigators: Factors affecting bleeding risk during anticoagulant therapy in patients with atrial fibrillation: observations from the Atrial Fibrillation Follow-up Investigation of Rhythm Management (AFFIRM) study. Am Heart J 2005, 149(4):650-656.

30. Dimberg I, Grzymala-Lubanski B, Hägerfelth A, Rosenqvist M, Svensson P Själander A: Computerised assistance for warfarin dosage-effects on treatment quality. Eur J Intern Med 2012, 23(8):742-744.

31. The European Atrial Fibrillation Trial Study Group: Optimal oral anticoagulant therapy in patients with nonrheumatic atrial fibrillation and recent cerebral ischemia. N Engl J Med 1995, 333(1):5-10.

32. Ellis DJ, Usman MH, Milner PG, Canafax DM, Ezekowitz MD: The first evaluation of a novel vitamin $\mathrm{K}$ antagonist, tecarfarin (ATI-5923), in patients with atrial fibrillation. Circulation 2009, 120(12):1029-1035. 2 p following 1035.

33. Evans A, Perez I, Yu G, Kalra L: Secondary stroke prevention in atrial fibrillation: lessons from clinical practice. Stroke 2000, 31(9):2106-2111.

34. Evans A, Perez I, Yu G, Kalra L: Should stroke subtype influence anticoagulation decisions to prevent recurrence in stroke patients with atrial fibrillation? Stroke 2001, 32(12):2828-2832.

35. Ezekowitz MD, Reilly PA, Nehmiz G, Simmers TA, Nagarakanti R, Parcham-Azad K, Pedersen KE, Lionetti DA, Stangier J, Wallentin L: Dabigatran with or without concomitant aspirin compared with warfarin alone in patients with nonvalvular atrial fibrillation (PETRO Study). Am J Cardiol 2007, 100(9):1419-1426.

36. Fang MC, Chang Y, Hylek EM, Rosand J, Greenberg SM, Go AS, Singer DE: Advanced age, anticoagulation intensity, and risk for intracranial hemorrhage among patients taking warfarin for atrial fibrillation. Ann Intern Med 2004, 141(10):745-752.

37. Gallagher AM, Setakis E, Plumb JM, Clemens A, van Staa TP: Risks of stroke and mortality associated with suboptimal anticoagulation in atrial fibrillation patients. Thromb Haemost 2011, 106(5):968-977. 
38. Garcia DA, Wallentin L, Lopes RD, Thomas L, Alexander JH, Hylek EM, Ansell J, Hanna M, Lanas F, Flaker G, Commerford P, Xavier D, Vinereanu D, Yang H, Granger CB: Apixaban versus warfarin in patients with atrial fibrillation according to prior warfarin use: results from the Apixaban for Reduction in Stroke and Other Thromboembolic Events in Atrial Fibrillation trial. Am Heart J 2013, 166(3):549-558.

39. Gladstone DJ, Bui E, Fang J, Laupacis A, Lindsay MP, Tu JV, Silver FL, Kapral MK Potentially preventable strokes in high-risk patients with atrial fibrillation who are not adequately anticoagulated. Stroke 2009, 40(1):235-240.

40. Go AS, Hylek EM, Chang Y, Phillips KA, Henault LE, Capra AM, Jensvold NG, Selby $\mathrm{JV}$, Singer DE: Anticoagulation therapy for stroke prevention in atrial fibrillation: how well do randomized trials translate into clinical practice? JAMA 2003, 290(20):2685-2692.

41. Gulløv AL, Koefoed BG, Petersen P, Pedersen TS, Andersen ED, Godtfredsen J, Boysen G: Fixed minidose warfarin and aspirin alone and in combination vs adjusted-dose warfarin for stroke prevention in atrial fibrillation: Second Copenhagen Atrial Fibrillation, Aspirin, and Anticoagulation Study. Arch Intern Med 1998, 158(14):1513-1521.

42. Gulløv AL, Koefoed BG, Petersen P: Bleeding during warfarin and aspirin therapy in patients with atrial fibrillation: the AFASAK 2 study. Atrial Fibrillation Aspirin and Anticoagulation. Arch Intern Med 1999, 159(12):1322-1328.

43. Gurwitz JH, Monette J, Rochon PA, Eckler MA, Avorn J: Atrial fibrillation and stroke prevention with warfarin in the long-term care setting. Arch Intern Med 1997, 157(9):978-984.

44. Hannon N, Callaly E, Moore A, Ní Chróinín D, Sheehan O, Marnane M, Merwick A, Kyne L, Duggan J, McCormack PM, Dolan E, Crispino-O'Connell G, Harris D, Horgan G, Williams D, Kelly PJ: Improved late survival and disability after stroke with therapeutic anticoagulation for atrial fibrillation: a population study. Stroke 2011, 42(9):2503-2508

45. Hart RG, Pearce LA, Asinger RW, Herzog CA: Warfarin in atrial fibrillation patients with moderate chronic kidney disease. Clin J Am Soc Nephrol 2011, 6(11):2599-2604

46. Heidinger KS, Bernardo A, Taborski U, Müller-Berghaus G: Clinical outcome of self-management of oral anticoagulation in patients with atrial fibrillation or deep vein thrombosis. Thromb Res 2000, 98(4):287-293.

47. Hellemons BS, Langenberg M, Lodder J, Vermeer F, Schouten HJ, Lemmens T, van Ree JW, Knottnerus JA: Primary prevention of arterial thromboembolism in non-rheumatic atrial fibrillation in primary care: randomised controlled trial comparing two intensities of coumarin with aspirin. BMJ 1999, 319(7215):958-964.

48. Ho LY, Siu CW, Yue WS, Lau CP, Lip GY, Tse HF: Safety and efficacy of oral anticoagulation therapy in Chinese patients with concomitant atrial fibrillation and hypertension. J Hum Hypertens 2011, 25(5):304-310.

49. Holmes DR, Reddy VY, Turi ZG, Doshi SK, Sievert H, Buchbinder M, Mullin CM Sick P, PROTECT AF Investigators: Percutaneous closure of the left atrial appendage versus warfarin therapy for prevention of stroke in patients with atrial fibrillation: a randomised non-inferiority trial. Lancet 2009 374(9689):534-542.

50. Hori M, Matsumoto M, Tanahashi N, Momomura S, Uchiyama S, Goto S, Izumi T, Koretsune Y, Kajikawa M, Kato M, Ueda H, Iwamoto K, Tajiri M, J-ROCKET AF study investigators: Rivaroxaban vs. warfarin in Japanese patients with atrial fibrillation - the J-ROCKET AF study -. Circ J 2012, 76(9):2104-2111.

51. Hylek EM, Go AS, Chang Y, Jensvold NG, Henault LE, Selby JV, Singer DE: Effect of intensity of oral anticoagulation on stroke severity and mortality in atrial fibrillation. N Engl J Med 2003, 349(11):1019-1026.

52. Hylek EM, Evans-Molina C, Shea C, Henault LE, Regan S: Major hemorrhage and tolerability of warfarin in the first year of therapy among elderly patients with atrial fibrillation. Circulation 2007, 115(21):2689-2696.

53. Jacobs LG, Billett HH, Freeman K, Dinglas C, Jumaquio L: Anticoagulation for stroke prevention in elderly patients with atrial fibrillation, including those with falls and/or early-stage dementia: a single-center, retrospective observational study. Am J Geriatr Pharmacother 2009, 7(3):159-166.

54. Jones M, McEwan P, Morgan CL, Peters JR, Goodfellow J, Currie CJ: Evaluation of the pattern of treatment, level of anticoagulation control, and outcome of treatment with warfarin in patients with non-valvar atrial fibrillation: a record linkage study in a large British population. Heart 2005, 91(4):472-477.
55. Kalra L, Yu G, Perez I, Lakhani A, Donaldson N: Prospective cohort study to determine if trial efficacy of anticoagulation for stroke prevention in atrial fibrillation translates into clinical effectiveness. BMJ 2000, 320(7244):1236-1239.

56. Kim JH, Song YB, Shin DH, Kim JS, Choi JO, On YK, Kim JS: How well does the target INR level maintain in warfarin-treated patients with non-valvular atrial fibrillation? Yonsei Med J 2009, 50(1):83-88.

57. Kim YK, Nieuwlaat R, Connolly SJ, Schulman S, Meijer K, Raju N, Kaatz S, Eikelboom JW: Effect of a simple two-step warfarin dosing algorithm on anticoagulant control as measured by time in therapeutic range: a pilot study. J Thromb Haemost 2010, 8(1):101-106.

58. Kulo A, Mulabegović N, Kusturica J, Hadzić H, Burnazović-Ristić L, Rakanović-Todić M, Mehmedović A, Lepara O: Outpatient management of oral anticoagulation therapy in patients with nonvalvular atrial fibrillation. Bosn J Basic Med Sci 2009, 9(4):313-319.

59. Lee SJ, Shin DH, Hwang HJ, Kim JY, Pak HN, Lee MH, Joung B: Bleeding risk and major adverse events in patients with previous ulcer on oral anticoagulation therapy. Am J Cardiol 2012, 110(3):373-377.

60. Malik AK, Taylor AJ: Can warfarin randomized trials be reproduced in 'real life'? Adherence to warfarin guidelines for intensity of anticoagulation in a university-based warfarin clinic. South Med J 2000 93(1):58-61

61. Mant J, Hobbs FD, Fletcher K, Roalfe A, Fitzmaurice D, Lip GY, Murray E, BAFTA investigators; Midland Research Practices Network (MidReC): Warfarin versus aspirin for stroke prevention in an elderly community population with atrial fibrillation (the Birmingham Atrial Fibrillation Treatment of the Aged Study, BAFTA): a randomised controlled trial. Lancet 2007, 370(9586):493-503.

62. Matchar DB, Samsa GP, Cohen SJ, Oddone EZ, Jurgelski AE: Improving the quality of anticoagulation of patients with atrial fibrillation in managed care organizations: results of the managing anticoagulation services trial. Am J Med 2002, 113(1):42-51.

63. McBride $D$, Brüggenjürgen $B$, Roll $S$, Willich SN: Anticoagulation treatment for the reduction of stroke in atrial fibrillation: a cohort study to examine the gap between guidelines and routine medical practice. J Thromb Thrombolysis 2007, 24(1):65-72.

64. McCormick D, Gurwitz JH, Goldberg RJ, Becker R, Tate JP, Elwell A, Radford MJ: Prevalence and quality of warfarin use for patients with atrial fibrillation in the long-term care setting. Arch Intern Med 2001, 161(20):2458-2463.

65. Melamed OC, Horowitz G, Elhayany A, Vinker S: Quality of anticoagulation control among patients with atrial fibrillation. Am J Manag Care 2011, 17(3):232-237

66. Menzin J, Boulanger L, Hauch O, Friedman M, Marple CB, Wygant G, Hurley JS, Pezzella S, Kaatz S: Quality of anticoagulation control and costs of monitoring warfarin therapy among patients with atrial fibrillation in clinic settings: a multi-site managed-care study. Ann Pharmacother 2005, 39(3):446-451

67. Morocutti C, Amabile G, Fattapposta F, Nicolosi A, Matteoli S, Trappolini M, Cataldo G, Milanesi G, Lavezzari M, Pamparana F, Coccheri S: Indobufen versus warfarin in the secondary prevention of major vascular events in nonrheumatic atrial fibrillation. SIFA (Studio Italiano Fibrillazione Atriale) Investigators. Stroke 1997, 28(5):1015-1021.

68. Neree C: Quality of oral anticoagulation in patients with atrial fibrillation: a cross-sectional study in general practice. Eur J Gen Pract 2006, 12(4):163-168

69. Nichol MB, Knight TK, Dow T, Wygant G, Borok G, Hauch O, O'Connor R: Quality of anticoagulation monitoring in nonvalvular atrial fibrillation patients: comparison of anticoagulation clinic versus usual care. Ann Pharmacother 2008, 42(1):62-70.

70. Njaastad AM, Abildgaard U, Lassen JF: Gains and losses of warfarin therapy as performed in an anticoagulation clinic. J Intern Med 2006, 259(3):296-304.

71. Nozawa T, Asanoi H, Inoue H, TOWARD Investigators, Toyama Warfarin RationalDosage: Instability of anticoagulation intensity contributes to occurrence of ischemic stroke in patients with non-rheumatic atrial fibrillation. Jpn Circ J 2001, 65(5):404-408.

72. Ogawa S, Shinohara Y, Kanmuri K: Safety and efficacy of the oral direct factor xa inhibitor apixaban in Japanese patients with non-valvular atrial fibrillation. -The ARISTOTLE-J study-. Circ J 2011, 75(8):1852-1859.

73. Okumura K, Komatsu T, Yamashita T, Okuyama Y, Harada M, Konta Y, Hatayama T, Horiuchi D, Tsushima E: Time in the therapeutic range during 
warfarin therapy in Japanese patients with non-valvular atrial fibrillation. A multicenter study of its status and infuential factors-. Circ J 2011, 75(9):2087-2094

74. Olsson SB, Executive Steering Committee of the SPORTIF III Investigators: Stroke prevention with the oral direct thrombin inhibitor ximelagatran compared with warfarin in patients with non-valvular atrial fibrillation (SPORTIF III): randomised controlled trial. Lancet 2003, 362(9397):1691-1698.

75. Ono A, Fujita T: Low-intensity anticoagulation for stroke prevention in elderly patients with atrial fibrillation: efficacy and safety in actual clinical practice. J Clin Neurosci 2005, 12(8):891-894.

76. Patel MR, Mahaffey KW, Garg J, Pan G, Singer DE, Hacke W, Breithardt G, Halperin JL, Hankey GJ, Piccini JP, Becker RC, Nessel CC, Paolini JF, Berkowitz SD, Fox KA, Califf RM, ROCKET AF Investigators: Rivaroxaban versus warfarin in nonvalvular atrial fibrillation. N Engl J Med 2011, 365(10):883-891.

77. Pengo V, Zasso A, Barbero F, Banzato A, Nante G, Parissenti L, John N, Noventa F, Dalla Volta S: Effectiveness of fixed minidose warfarin in the prevention of thromboembolism and vascular death in nonrheumatic atrial fibrillation. Am J Cardiol 1998, 82(4):433-437.

78. Pengo V, Legnani C, Noventa F, Palareti G, ISCOAT Study Group. (Italian Study on Complications of Oral Anticoagulant Therapy): Oral anticoagulant therapy in patients with nonrheumatic atrial fibrillation and risk of bleeding. A Multicenter Inception Cohort Study. Thromb Haemost 2001, 85(3):418-422.

79. Pengo V, Cucchini U, Denas G, Davidson BL, Marzot F, Jose SP, lliceto S: Lower versus standard intensity oral anticoagulant therapy (OAT) in elderly warfarin-experienced patients with non-valvular atrial fibrillation. Thromb Haemost 2010, 103(2):442-449.

80. Pérez-Gómez F, Alegría E, Berjón J, Iriarte JA, Zumalde J, Salvador A, Mataix L, NASPEAF Investigators: Comparative effects of antiplatelet, anticoagulant, or combined therapy in patients with valvular and nonvalvular atrial fibrillation: a randomized multicenter study. J Am Coll Cardiol 2004, 44(8):1557-1566.

81. Poli D, Antonucci E, Marcucci R, Fatini C, Alterini B, Mannini L, Falciani M, Abbate R, Gensini GF, Prisco D: Risk of bleeding in very old atrial fibrillation patients on warfarin: relationship with ageing and CHADS2 score. Thromb Res 2007, 121(3):347-352.

82. Poli D, Antonucci E, Grifoni E, Abbate R, Gensini GF, Prisco D: Bleeding risk during oral anticoagulation in atrial fibrillation patients older than 80 years. J Am Coll Cardiol 2009, 54(11):999-1002.

83. Poli D, Antonucci E, Marcucci R, Mannini L, Falciani M, Abbate R, Gensini GF, Prisco D: The quality of anticoagulation on functional outcome and mortality for TIA/stroke in atrial fibrillation patients. Int J Cardiol 2009, 132(1):109-113.

84. Poli D, Antonucci E, Grifoni E, Abbate R, Gensini GF, Prisco D: Gender differences in stroke risk of atrial fibrillation patients on oral anticoagulant treatment. Thromb Haemost 2009, 101(5):938-942.

85. Poller L, Keown M, Ibrahim S, Lowe G, Moia M, Turpie AG, Roberts C, van den Besselaar AM, van der Meer FJ, Tripodi A, Palareti G, Shiach C, Bryan S, Samama M, Burgess-Wilson M, Heagerty A, Maccallum P, Wright D, Jespersen J: An international multicenter randomized study of computer-assisted oral anticoagulant dosage vs. medical staff dosage. J Thromb Haemost 2008, 6(6):935-943.

86. Sadanaga T, Sadanaga M, Ogawa S: Evidence that D-dimer levels predict subsequent thromboembolic and cardiovascular events in patients with atrial fibrillation during oral anticoagulant therapy. J Am Coll Cardiol 2010, 55(20):2225-2231

87. Samsa GP, Matchar DB, Goldstein LB, Bonito AJ, Lux LJ, Witter DM, Bian J: Quality of anticoagulation management among patients with atrial fibrillation: results of a review of medical records from 2 communities. Arch Intern Med 2000, 160(7):967-973.

88. Schwammenthal Y, Bornstein N, Schwammenthal E, Schwartz R, Goldbourt U, Tsabari R, Koton S, Grossman E, Tanne D: Relation of effective anticoagulation in patients with atrial fibrillation to stroke severity and survival (from the National Acute Stroke Israeli Survey [NASIS]). Am J Cardiol 2010, 105(3):411-416.

89. Sconce $\mathrm{E}$, Avery P, Wynne H, Kamali F: Vitamin K supplementation can improve stability of anticoagulation for patients with unexplained variability in response to warfarin. Blood 2007, 109(6):2419-2423.

90. Shen AY, Yao JF, Brar SS, Jorgensen MB, Chen W: Racial/ethnic differences in the risk of intracranial hemorrhage among patients with atrial fibrillation. J Am Coll Cardiol 2007, 50(4):309-315.
91. Singer DE, Chang Y, Fang MC, Borowsky LH, Pomernacki NK, Udaltsova N, Go AS: Should patient characteristics influence target anticoagulation intensity for stroke prevention in nonvalvular atrial fibrillation?: the ATRIA study. Circ Cardiovasc Qual Outcomes 2009, 2(4):297-304.

92. Stroke Prevention in Atrial Fibrillation Investigators: Adjusted-dose warfarin versus low-intensity, fixed-dose warfarin plus aspirin for high-risk patients with atrial fibrillation: stroke prevention in atrial fibrillation III randomised clinical trial. Lancet 1996, 348(9028):633-638.

93. Sullivan RM, Zhang J, Zamba G, Lip GY, Olshansky B: Relation of gender-specific risk of ischemic stroke in patients with atrial fibrillation to differences in warfarin anticoagulation control (from AFFIRM). Am J Cardiol 2012, 110(12):1799-1802.

94. Tincani E, Baldini P, Crowther MA, Zanasi A, Ferrari P, Cenci AM, Rioli G: Bleeding rates in patients older than 90 years of age on vitamin $\mathrm{K}$ antagonist therapy for nonvalvular atrial fibrillation. Blood Coagul Fibrinolysis 2009, 20(1):47-51.

95. Van Spall HG, Wallentin L, Yusuf S, Eikelboom JW, Nieuwlaat R, Yang S, Kabali C, Reilly PA, Ezekowitz MD, Connolly SJ: Variation in warfarin dose adjustment practice is responsible for differences in the quality of anticoagulation control between centers and countries: an analysis of patients receiving warfarin in the randomized evaluation of long-term anticoagulation therapy (RE-LY) trial. Circulation 2012 126(19):2309-2316

96. Völler H, Glatz J, Taborski U, Bernardo A, Dovifat C, Heidinger K: Self-management of oral anticoagulation in nonvalvular atrial fibrillation (SMAAF study). Z Kardiol 2005, 94(3):182-186.

97. Walker GA, Heidenreich PA, Phibbs CS, Go AS, Chiu VY, Schmitt SK, Ananth L, Frayne SM: Mental illness and warfarin use in atrial fibrillation. Am J Manag Care 2011, 17(9):617-624.

98. Wieloch M, Själander A, Frykman V, Rosenqvist M, Eriksson N, Svensson PJ: Anticoagulation control in Sweden: reports of time in therapeutic range, major bleeding, and thrombo-embolic complications from the national quality registry AuriculA. Eur Heart J 2011, 32(18):2282-2289.

99. Weitz Jl, Connolly SJ, Patel I, Salazar D, Rohatagi S, Mendell J, Kastrissios H, Jin J, Kunitada S: Randomised, parallel-group, multicentre, multinational phase 2 study comparing edoxaban, an oral factor Xa inhibitor, with warfarin for stroke prevention in patients with atrial fibrillation. Thromb Haemost 2010, 104(3):633-641.

100. Wyse DG, Waldo AL, DiMarco JP, Domanski MJ, Rosenberg Y, Schron EB, Kellen JC, Greene HL, Mickel MC, Dalquist JE, Corley SD, Atrial Fibrillation Follow-up Investigation of Rhythm Management (AFFIRM) Investigators: A comparison of rate control and rhythm control in patients with atrial fibrillation. N Engl J Med 2002, 347(23):1825-1833.

101. Yamaguchi T: Optimal intensity of warfarin therapy for secondary prevention of stroke in patients with nonvalvular atrial fibrillation : a multicenter, prospective, randomized trial. Japanese Nonvalvular Atrial Fibrillation-Embolism Secondary Prevention Cooperative Study Group. Stroke 2000, 31(4):817-821.

102. Yasaka M, Minematsu K, Yamaguchi T: Optimal intensity of international normalized ratio in warfarin therapy for secondary prevention of stroke in patients with non-valvular atrial fibrillation. Intern Med 2001, 40(12):1183-1188

103. Yousef ZR, Tandy SC, Tudor V, Jishi F, Trent RJ, Watson DK, Cowell RP. Warfarin for non-rheumatic atrial fibrillation: five year experience in a district general hospital. Heart 2004, 90(11):1259-1262.

104. Chiquette $\mathrm{E}$, Amato MG, Bussey HI: Comparison of an anticoagulation clinic with usual medical care: anticoagulation control, patient outcomes, and health care costs. Arch Intern Med 1998, 158(15):1641-1647.

105. Witt DM, Sadler MA, Shanahan RL, Mazzoli G, Tillman DJ: Effect of a centralized clinical pharmacy anticoagulation service on the outcomes of anticoagulation therapy. Chest 2005, 127:1515-1522.

106. Garwood CL, Dumo P, Baringhaus SN, Laban KM: Quality of anticoagulation care in patients discharged from a pharmacist-managed anticoagulation clinic after stabilization of warfarin therapy. Pharmacotherapy 2008, 28(1):20-26

107. Nelson WW, Damaraju CV, Lu L, Schein J, Fields LE, Wildgoose P, Chang P: Patterns of INR stability among newly initiated warfarin patients with NVAF, Annual Meeting of the American Heart Association Scientific Sessions. Dallas TX: Conference Presentation. Dallas TX: 2013.

108. Laliberté F, Cloutier M, Nelson WW, Coleman Cl, Pilon D, Olson WH, Damaraju CV, Schein JR, Lefebvre P: Real-world comparative effectiveness 
and safety of rivaroxaban and warfarin in nonvalvular atrial fibrillation patients. Curr Med Res Opin 2014, [Epub ahead of print]

109. Nutescu EA, Bathija S, Sharp LK, Gerber BS, Schumock GT, Fitzgibbon ML: Anticoagulation patient self-monitoring in the United States: considerations for clinical practice adoption. Pharmacotherapy 2011, 31(12):1161-1174.

doi:10.1186/1477-9560-12-14

Cite this article as: Mearns et al:: Quality of vitamin K antagonist control and outcomes in atrial fibrillation patients: a meta-analysis and meta-regression. Thrombosis Journal 2014 12:14.

\section{Submit your next manuscript to BioMed Central and take full advantage of:}

- Convenient online submission

- Thorough peer review

- No space constraints or color figure charges

- Immediate publication on acceptance

- Inclusion in PubMed, CAS, Scopus and Google Scholar

- Research which is freely available for redistribution 